\title{
Exploiting Molecular Self-Assembly: From Urea-Based Organocatalysts to Multifunctional Supramolecular Gels
}

\author{
Eva-Maria Schön, ${ }^{[a]}$ Eugenia Marqués-López, ${ }^{[b]}$ Raquel P. Herrera, ${ }^{* b]}$ Carlos Alemán, ${ }^{[c]}$ and David \\ Díaz Díaz ${ }^{\text {[a,d] }}$
}

\begin{abstract}
In this work we describe the self-assembly properties of chiral $N, N^{\prime}$ disubstituted urea-based organocatalyst 1 that lead to the formation of stable hierarchical supramolecular gels in organic solvents at concentrations ranging from 3 to $50 \mathrm{~g} \mathrm{~L}^{-1}$. The major driving forces for the gelation phenomenon are hydrogen bonding and $\pi-\pi$ interactions according to FT-IR, ${ }^{1} \mathrm{H}$ NMR and computational studies. The gelation scope could be interpreted based on Kamlet-Taft solvatochromic parameters. TEM, SEM and AFM imaging revealed that a variety of morphologies including helical, laths,
\end{abstract}

porous and lamellar nanostructures could be obtained by varying the solvent. Experimental gelation tests and computational structural analysis of various structurally related compounds proved the existence of a unique set of molecular interactions and an optimal hydrophilic/hydrophobic balance in $\mathbf{1}$ that drive the formation of stable gels. Responses to thermal, mechanical, optical and chemical stimuli, as well as multifunctionality were demonstrated in some model gel materials. Specifically, 1 could be used for the phase selective gelation of organic solvent/water mixtures. In addition, the gel prepared in glycerol was found to be thixotropic and provided a sensitive colorimetric method for the detection of $\mathrm{Ag}(\mathrm{I})$ ions at millimolar concentrations in aqueous solution. Furthermore, the gel matrix obtained in toluene served as nanoreactor for the synthesis of dispersed gold nanoparticles (average size $\sim 15 \mathrm{~nm}$ ) and for the Friedel-Crafts alkylation of $1 H$-indole with trans- $\beta$ nitrostyrene.

Keywords: ureas $\bullet$ self-assembly $\bullet$
organogels $\bullet$ multistimuli
responsiveness $\bullet$ multifunctional
materials

\section{Introduction}

Multifunctional stimuli-responsive structures have drawn great attention in the last decade due to their potential use in advanced devices and help to expand fundamental scientific understanding. ${ }^{[i]}$ Such systems possess properties that allow them to perform more than one function in a device or material where interfacial properties are coupled. Inspired by abundant examples in nature where multifunctionality is a norm (e.g., multifunctional extracellular matrices), the main need for the development of multifunctional materials is that specific problems cannot be always solved by the mere combination of materials with different functions due to critical technological barriers.

[a] Dr. E.-M. Schön, Prof. D. D. Díaz

Institut für Organische Chemie, Universität Regensburg

Universitätsstr. 31, 93053 Regensburg (Germany)

David.Diaz@chemie.uni-regensburg.de

[b] Dr. E. Marqués-López, Dr. R. P. Herrera

Departamento de Química Orgánica. Instituto de Síntesis Química y Catálisis Homogénea (ISQCH), CSIC-Universidad de Zaragoza Pedro Cerbuna 12, 50009 Zaragoza (Spain)

raquelph@unizar.es

[c] Prof. C. Alemán

Departament d'Enginyeria Química, ETSEIB, Universitat Politècnica de Catalunya, Av. Diagonal 647, 08028 Barcelona (Spain)

[d] IQAC-CSIC, Jordi Girona 18-26, 08034 Barcelona (Spain)

Supporting information for this article is available on the WWW under http://www.chemeurj.org/ or from the author.
In the above context, self-assembled gels ${ }^{[i i]}$ constitute promising candidates to achieve multifunctional materials for different applications. ${ }^{[i i i]}$ In contrast to chemical gels, ${ }^{[i v]}$ which are based on covalent bonds, physical or supramolecular gels ${ }^{[\mathrm{v}]}$ are made of either low-molecular-weight (LMW) compounds or polymers through non-covalent interactions (e.g., hydrogen bonding, $\pi-\pi$ stacking) that usually provide a reversible response to external stimuli (e.g., gel-to-sol thermal transition). In general, the solid-like appearance of gels derived from a very efficient entrapment of the solvent molecules, usually by capillary forces, ${ }^{[\mathrm{vi}]}$ into the interstices of a solid matrix with high surface area formed upon the entanglement of 1D supramolecular fibrilar assemblies. There is an extensive collection of functional moieties that can induce the formation of such assemblies in solution. ${ }^{[\mathrm{v}]}$ Among those, the ureide group is one of the best-known hydrogen bonding functional groups, which has been widely used to fabricate valuable supramolecular architectures, ${ }^{\text {[vii] }}$ including gel networks based on mono- or polyurea gelators, ${ }^{[\text {viii] }}$ via a directional assembly process.

Herein, we report and rationalize the self-assembly properties of a known urea-based organocatalyst that lead to the formation of multifunctional and multiresponsive supramolecular gels in organic solvents. 


\section{Results and Discussion}

Design and synthesis of compounds: During our research programs focused on both the development of new organocatalysts and the use of unconventional reaction media (e.g., softgel materials, ionic liquids), we paid close attention to the tendency of the known $N, N^{\prime}$-disubstituted urea-based organocatalyst $(+)-\mathbf{1}^{[9]}$ (Scheme 1) to increase the viscosity of some common organic solvents leading to the in situ formation of jelly-like lumps during its synthesis. We decided to investigate in detail the gelation ability of $\mathbf{1}$ after taking into consideration the facile and scalable synthesis of this type of compounds, their intrinsic potential as multifunctional molecules and the previous studies reported so far on urea-based organogelators. ${ }^{[8]}$ Compound $\mathbf{1}$ is easily accessible by an equimolar reaction of commercial $(1 S, 2 R)$-1-amino-2,3-dihydro-1 $H$-inden-2-ol and 3,5-bis(trifluoromethyl)phenyl isocyanate in methylene chloride at room temperature (Scheme 1).
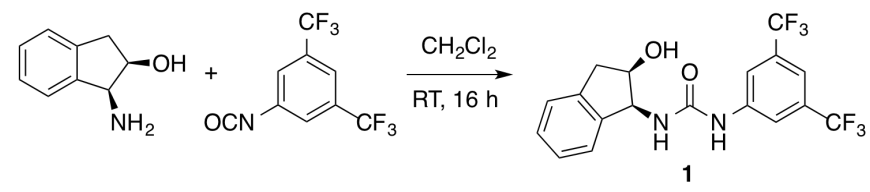

Scheme 1. Synthesis of $N, N^{\prime}$-disubstituted urea (+)-1.

In order to correlate the structural features of $\mathbf{1}$ with the gelation properties, we designed and synthesized a library of analogous compounds 2-7 (Figure 1) following a similar synthetic procedure. The structural complexity of $\mathbf{1}$ was greatly reduced by replacing both aromatic residues by 3,5-bis(trifluoromethyl)phenyl groups (compound 3) or phenyl groups (compound 4). To study the influence of the stereochemical configuration of the stereogenic centers we also carried out the synthesis of the diastereomer $\mathbf{5}$. The evident intramolecular hydrogen bonding between the carbonyl group and the hydroxyl group at the 2,3-dihydro- $1 H$-indene residue inspired us to prepare compound $\mathbf{6}$ lacking the hydroxy group and thiourea derivative 2. Additionally, we considered the removal of only the bulky trifluoromethyl groups (compound 7) since they are also known to lower the basicity and/or confer distinctive solvation properties of organic compounds, which play a key role on the gelation phenomena. ${ }^{[2-6]}$

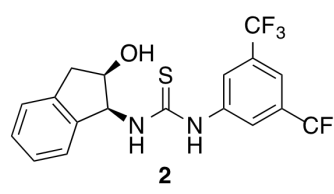<smiles>O=C(Nc1ccccc1)Nc1ccccc1</smiles>

4

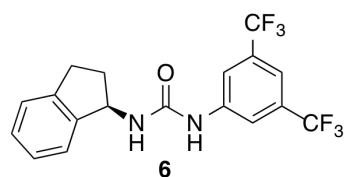<smiles>O=C(Nc1cc(C(F)(F)F)cc(C(F)(F)F)c1)Nc1cc(C(F)(F)F)cc(C(F)(F)F)c1</smiles><smiles>O=C(Nc1cc(C(F)(F)F)cc(C(F)(F)F)c1)N[C@H]1c2ccccc2CC1O</smiles>

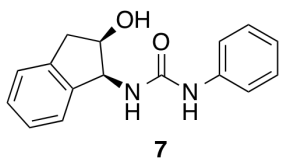

Figure 1. Library of additional compounds 2-7 used in this work.

All synthesized compounds were satisfactorily characterized after purification by silica gel column chromatography. ${ }^{[10]}$
Gelation ability and gel stability: The gelation ability of urea $\mathbf{1}$ was first evaluated for 33 different solvents using the classical heating-cooling process within a broad concentration range. The state of the resulting mixture was initially examined by the "stableto-inversion of a test tube" method. After the visual inspection, the viscoelastic gel nature of those samples showing no gravitational flow upon turning the vial upside-down was further confirmed by oscillatory rheological measurements in model solvents (vide infra).

We were delighted to observe that compound 1 induced gelation of 14 solvents at a critical gelation concentration (CGC) between 3 and $50 \mathrm{~g} \mathrm{~L}^{-1}$ (Table 1). These values imply the immobilization of $10^{2}-10^{3}$ (order of magnitude) solvent molecules per gelator molecule. In most cases, complete gelation was achieved within $5 \mathrm{~min}$ and $1 \mathrm{~h}$. A clear preference for gelation of aromatic (entries 1-9) and chlorinated solvents (entries 11-13) was observed. All organogels obtained at the CGC were transparent except in nitrobenzene, which was completely yellowish opaque indicating the formation of aggregates smaller than the visible wavelength range $(\lambda=400-700$ $\mathrm{nm}$ ) (Figure 2). Such optical differences highlight the importance of the interactions between solvent and gelator molecules for the growth and stabilization of the supramolecular network.

Table 1. Gelation scope of $\mathbf{1}$, optical appearance $(\mathrm{OA})$ of the gels, critical gelation concentrations (CGC), gelation times and gel-to-sol transition temperatures $\left(T_{\text {gel }}\right){ }^{[\mathrm{a}]}$

\begin{tabular}{|c|c|c|c|c|c|c|}
\hline Entry & Solvent & Phase & $\mathrm{OA}$ & $\begin{array}{l}\mathrm{CGC} \\
{\left[\mathrm{g} \mathrm{L}^{-1}\right]}\end{array}$ & $\begin{array}{l}\text { Gelatin } \\
\text { time }\end{array}$ & $\begin{array}{l}T_{\text {gel }}{ }^{[\mathrm{d}]} \\
{\left[{ }^{\circ} \mathrm{C}\right]}\end{array}$ \\
\hline 1 & Toluene & G & $\mathrm{T}$ & 3 & $30 \pm 5 \mathrm{~min}$ & 55 \\
\hline 2 & Benzene & G & $\mathrm{T}$ & 3 & $30 \pm 5 \min$ & 41 \\
\hline 3 & Chlorobenzene & G & $\mathrm{T}$ & 3.6 & $7 \pm 2 \mathrm{~min}$ & 54 \\
\hline 4 & 1,2-Dichlorobenzene & G & $\mathrm{T}$ & 3.6 & $7 \pm 2 \mathrm{~min}$ & 62 \\
\hline 5 & 1,3-Dichlorobenzene & G & $\mathrm{T}$ & 3.6 & $7 \pm 2 \min$ & 64 \\
\hline 6 & Mesitylene & G & $\mathrm{T}$ & 3.5 & $5 \pm 1 \mathrm{~h}$ & nd \\
\hline 7 & $o$-Xylene & G & $\mathrm{T}$ & 3.5 & $8 \pm 2 \mathrm{~h}$ & nd \\
\hline 8 & $m$-Xylene & G & $\mathrm{T}$ & 3.5 & $5 \pm 1 \mathrm{~h}$ & nd \\
\hline 9 & Nitrobenzene & G & $\mathrm{O}$ & 50 & $30 \pm 5 \mathrm{~min}$ & 41 \\
\hline 10 & Glycerol $^{[b]}$ & $\mathrm{G}^{[\mathrm{c}]}$ & $\mathrm{T}$ & 3 & $40 \pm 10 \mathrm{~min}$ & 74 \\
\hline 11 & Methylene chloride & G & $\mathrm{T}$ & 4 & $20 \pm 5 \mathrm{~min}$ & 46 \\
\hline 12 & Chloroform & G & $\mathrm{T}$ & 7 & $45 \pm 10 \mathrm{~min}$ & nd \\
\hline 13 & Carbon tetrachloride & G & $\mathrm{T}$ & 5 & $10 \pm 5 \min$ & 84 \\
\hline 14 & Nitromethane & $\mathrm{G}^{[\mathrm{c}]}$ & $\mathrm{T}$ & 25 & $24 \mathrm{~h}$ & nd \\
\hline
\end{tabular}

[a] Gels obtained after heating-cooling cycle. Volume $=1 \mathrm{~mL}$. Abbreviations: $\mathrm{G}=$ gel; $\mathrm{T}=$ transparent gel; $\mathrm{O}=$ opaque gel; nd $=$ not determined due to gel weakness. [b] Commercial sample contained $10 \mathrm{wt} . \%$ water. [c] A minor fraction of insoluble material remained. [d] Values calculated by the inverse flow method. Estimated error $\pm 2{ }^{\circ} \mathrm{C}$.

Compound 1 was found to be insoluble in both water and $n$-heptane upon heating and/or extensive sonication $(2 \mathrm{~h})$, whereas stable clear solutions were obtained at $50 \mathrm{~g} \mathrm{~L}^{-1}$ upon heating-cooling in acetonitrile, ethyl acetate, ethanol, methanol, dimethyl sulfoxide, 
$\mathrm{N}, \mathrm{N}$-dimethylformamide, dimethylacetamide, tetrahydrofuran, diethyl ether, 1,2-dimethoxyethane, acetone, cyclohexanone, 3methyl-butan-2-one, methyl tert-butyl ether, 1,4-dioxane, benzonitrile and rapeseed oil. Exceptionally, gels in nitromethane (entry 14) and 90 wt.\% glycerol (entry 10 ) could be also obtained. The case of glycerol is particularly interesting since a) it was the only alcoholic solvent where gelation was successful and b) it is a non-toxic, non-hazardous, non-volatile, and biodegradable solvent widely used in manifold industries including, among others, food, anti-freeze, pharmaceutical and personal care applications. ${ }^{[11]}$

Based on the solvent parameters (vide infra) and in order to optimize the number of experiments, we chose three representative model solvents (i.e., $\mathrm{CH}_{2} \mathrm{Cl}_{2}$, toluene and glycerol), among those gelled by $\mathbf{1}$, to conduct comparative gelation experiments with the structurally related compounds 2-7. Very interestingly, the analogous 2-6 did not show any gelation ability in the model solvents. Only compound 7, lacking the trifluoromethyl groups, was able to form a transient weak gel in toluene at a concentration of $3 \mathrm{~g}$ $\mathrm{L}^{-1}$, and a stable gel in glycerol at $5 \mathrm{~g} \mathrm{~L}^{-1}$. Compound 7 required not only a 5-fold higher concentration than $\mathbf{1}$ to form a steady homogeneous gel in glycerol, but also ca. 5-fold longer gelation time. These results clearly suggested the existence of unique interand/or intramolecular interactions as well as an optimum balance between hydrophobic and hydrophilic domains that largely favor the spontaneous self-assembly of $\mathbf{1}$ in solution leading to supramolecular aggregates with a lifetime long enough to allow their anisotropic growth and consequent stable gel formation.
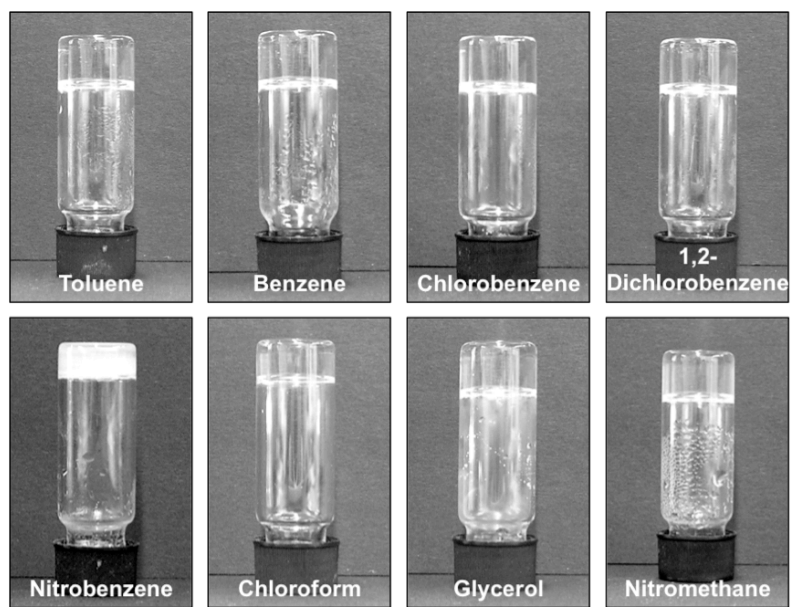

Figure 2. Representative digital photographs of upside-down vials containing organogels made of $\mathbf{1}$ in different solvents at the CGC as shown in Table 1.

It is worth to mention that all our attempts to obtain isotropic solutions of potential gelators in the model solvents and subsequent formation of stable gels using either (a) sonication instead of the heating-cooling cycle, or (b) predissolving the compound in the minimum amount of a non-protic polar solvent such as DMSO followed by addition of the testing solvent (e.g., maintaining the CGC value as given in Table 1) at room temperature were fruitless.

Thermal and temporal stability: The organogels were found to be thermoreversible and stable to multiple heating-cooling cycles without any detriment on the gelation ability and gel properties. The gel-to-sol transition temperatures $\left(T_{\text {gel }}\right)$ of all organogels were determined by the inverse flow method. ${ }^{[12]}$ As this method depends on the cooling rate and thermal history, among other factors, the values were correlated for model examples with the first endothermic transition observed by differential scanning calorimetry (DSC) (Figure S5). Consistently with the formation of more entwined networks, $T_{\text {gel }}$ increased considerably until reaching a plateau with increasing the concentration of the urea gelator 1 (e.g., $\Delta T_{\text {gel }}$ (toluene) $\sim 38^{\circ} \mathrm{C}$ upon increasing 2.3 -fold the concentration defined by the CGC, Figure 3). Interestingly, we found that the amount of water present in commercial 90 wt.\% glycerol was necessary to prepare the isotropic solution of the gelator and subsequent gels. Attempts to dissolve 1 in 99 wt.\% glycerol upon heating were unsuccessful. The $T_{\text {gel }}$ values increased considerably from 60 wt. $\%$ to 90 wt.\% glycerol (e.g., $64{ }^{\circ} \mathrm{C}$ at 70 wt. $\%$ and $74{ }^{\circ} \mathrm{C}$ at 90 wt.\%. The gels prepared with 60 wt. $\%$ glycerol were too fragile to resist inversion of the vial).

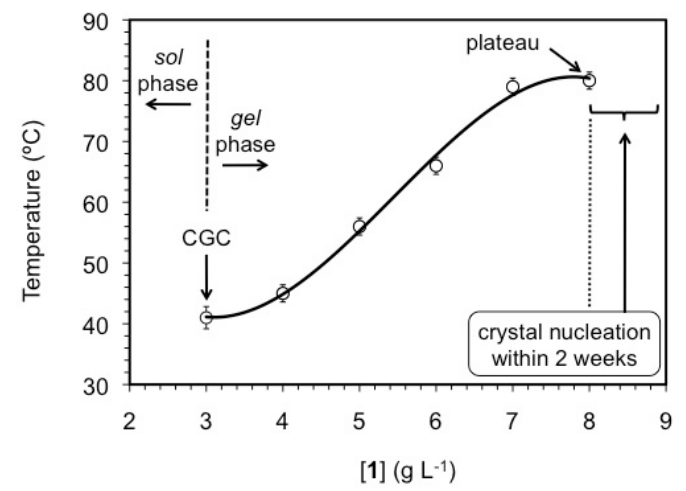

Figure 3. Phase diagram and evolution of $T_{\text {gel }}$ as a function of the concentration of gelator $\mathbf{1}$ in toluene.

Organogels made of $\mathbf{1}$ at the CGC remained stable for at least one month when stored undisturbed at room temperature. After this period, optical microscopic imaging of some materials revealed a very slow crystal growth (Figure 4), which clearly underlines the thermodynamic equilibrium between gel and crystalline phases. ${ }^{[13]}$ Nevertheless, the robustness of the gel network permitted its coexistence with the crystal nucleation for several months while remaining stable to the inversion of the vial. As expected, the crystallization kinetics also increased with gelator concentration.
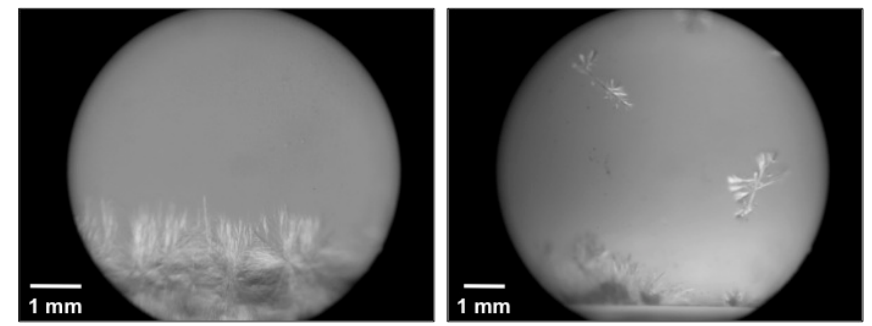

Figure 4. Optical microscope picture showing crystal formation in the gel matrix made of 1 at CGC (left) and at $15 \mathrm{~g} \mathrm{~L}^{-1}$ (right) after 5 weeks.

Influence of enantiomeric purity: As chirality plays a key role in the formation of gels, ${ }^{[14]}$ we investigated the gelation ability of $\mathbf{1}$ prepared at different enantiomeric excess by mixing appropriate amounts of the pure enantiomers (+)-1 and (-)-1. Stable gels upon inversion of the vials were only obtained when the enantiomerically pure urea gelator was used (Figure 5 and Figure S14). As expected, $(+)-1$ and (-)-1 showed identical gelation properties. Precipitation or small pieces of jelly-like aggregates were observed when the urea 
compound was used with enantiomeric excess (ee) below $80 \%$. The material made from the urea with $80 \%$ ee consisted in a mixture of precipitate and gel (gelation time in this case was double than using pure 1) and could support the inversion of the vial. However, after $48 \mathrm{~h}$ the material collapsed and only the sample made with $100 \%$ ee remained homogeneous, transparent and stable to the inversion of the vial.

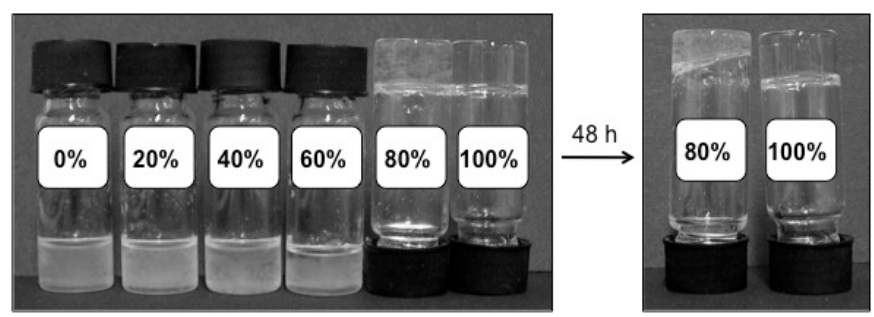

Figure 5. Influence of enantiomeric purity on the gelation ability of $\mathbf{1}$. Percent values indicate the enantiomeric excess of $\mathbf{1}$ used in each case.

Phase-selective gelation ability: Selective organogelation from organic solvent/water mixtures is an important task in environmental remediation. ${ }^{[15]}$ This ability has been reported for some efficient LMW organogelators, ${ }^{[15,16]}$ albeit it is still an uncommon feature in the area of supramolecular gels. Interestingly, water-insoluble urea 1 also showed this ability with a broad scope of organic solvents. Typically, a 1:1 v/v mixture containing water and any waterimmiscible organic solvent from Table 1 was heated and vigorously shaken in the presence of $\mathbf{1}$ at the corresponding CGC. After cooling down the homogeneous dispersion to room temperature, the organic phase was entirely gelled whereas the water phase remained liquid. Depending on the density of the organic phase, the gel material was located either above or below the water layer. For the latter case, the gel was stiff enough to hold the upper water phase upon inversion of the vial (Figure 6A). Both phases could be further separated by simple decantation or filtration. The thermal stability of the gel phase remained very similar to the gel obtained from the pure organic solvent (i.e., $\Delta T_{\text {gel }}$ ca. $\pm 5^{\circ} \mathrm{C}$ ).

Moreover, the phase selective gelation could be 50 -fold scaled up without any difficulty (Figure 6B). When the aqueous phase was stained with Evans Blue the organic phase remained clear upon gelation, indicating no diffusion of water through the interface. This is also understandable if we consider the intrinsic hydrophobicity of polyfluorinated 1 (e.g., water contact angle $(\mathrm{WCA}) \sim 110^{\circ}$ ), which is even enhanced upon the formation of the nanostructured gel network as a result of combining the low surface energy with a superior roughness (e.g., WCA of the xerogel obtained from the gel made in toluene $\left.\sim 140^{\circ}\right) .{ }^{[17]}$ Moreover, we observed that the model gels remained stable in the presence of water, $\mathrm{NaOH}(0.1 \mathrm{M})$ or even $\mathrm{HCl}(0.1 \mathrm{M})$ aqueous solutions (the experiments were carried out by placing $1 \mathrm{~mL}$ of the test solution on top of $1 \mathrm{~mL}$ of gel material).
A)
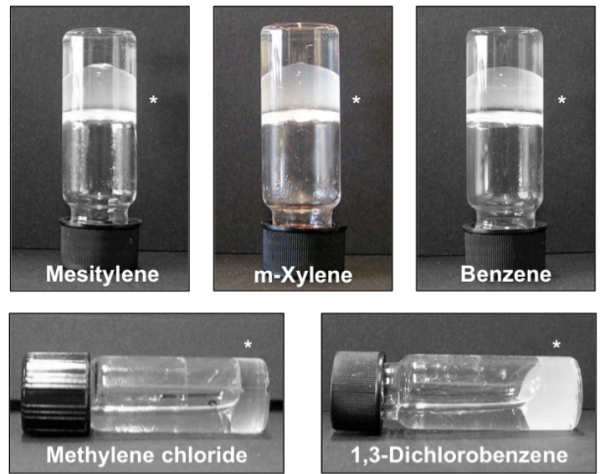

B)

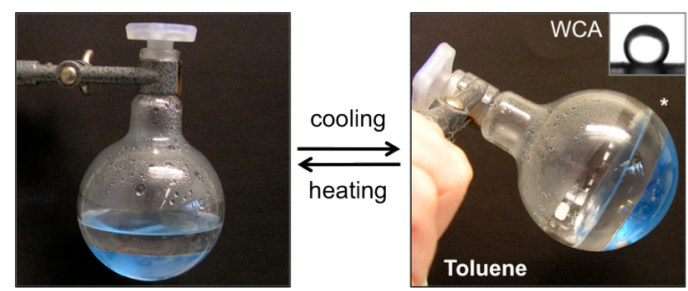

Figure 6. A) Representative digital photographs of phase selective gelation of organic solvent/water mixtures (total volume $=2 \mathrm{~mL}$ ). The organic solvent is marked with an asterisk. B) High scale phase selective gelation of 1:1 v/v toluene/water mixture (total volume $=0.1 \mathrm{~L}^{-1}$ ). Water-soluble T-1824 dye (Evans Blue) was used to differentiate better both phases. The aqueous phase remained completely liquid after gelation of the upper organic phase as evidenced by usual spinning of the magnetic stir bar. Inset on the right picture: Photograph of a water drop on a thin film of the gel made in toluene.

Correlation between gelation ability and solvent parameters: In order to rationalize the organogel formation we built and compared 3D plots according to the Kamlet-Taft solvatochromic parameters (i.e., hydrogen bond donor ability $(\alpha)$, hydrogen bond acceptor $(\beta)$, and polarizability $\left.\left(\pi^{*}\right)\right)^{[18]}$ and the Hansen solubility parameters (i.e. dispersive interactions $\left(\delta_{\mathrm{d}}\right)$, dipolar interactions $\left(\delta_{\mathrm{p}}\right)$ and hydrogen bonding $\left(\delta_{h}\right)$ interactions) (Table S1). ${ }^{[19]}$ The Kamlet-Taft solvent parameters have been associated to the ability of forming hydrogen bonded gels ( $\alpha$ value), thermal stability of the networks ( $\beta$ value) and stabilization of charges and dipoles during the gelation process $\left(\pi^{*}\right.$ value). ${ }^{[20]}$ With the only exception of glycerol, these parameters clearly delimited a gelation cuboid space (ca. 0.045 cubic units) defined by the following approximate dimensions: $0<\beta<0.3,0<$ $\alpha<0.2$ and $0.25<\pi^{*}<1$ (Figure 7). These limits indicate that $\pi^{*}$ has the lower influence in the formation of gels, whereas having relatively low and balanced hydrogen bond donor and acceptor abilities is critical. On the other hand, Hansen solubility parameters also provided an acceptable gelation space albeit only ca. $70 \%$ of the gelled solvents were found inside the cuboid space defined approximately by $0<\delta_{\mathrm{p}}<9,17.5<\delta_{\mathrm{d}}<20$ and $0.5<\delta_{\mathrm{h}}<6$ (Figure $\mathrm{S} 3$ ). Thus, dipolar interactions seem to be the most critical ones for a Hansen model, although a model based on the Kamlet-Taft parameters is comparatively more adequate for developing a gelation model in our case. No significant tendencies were observed for the gel properties (i.e., $\mathrm{CGC}, T_{\mathrm{gel}}$ ) in function of the individual solvent parameters. 


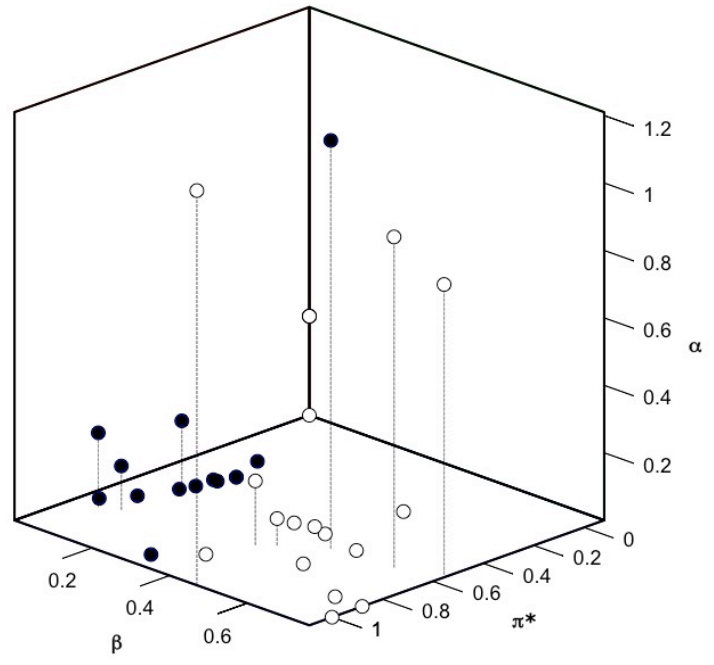

Figure 7. 3D scattering plot showing the results of the gelation tests and the Kamlet-Taft parameters of each solvent. Filled circles $=$ gelated solvents; open circles $=$ non-gelated solvents.

\section{Driving force study and computer modeling:}

FT-IR measurements: In agreement with other urea-based gelators, comparison of the FT-IR spectra of model xerogels prepared from the corresponding organogels by freeze-drying with those in solution phase and $\mathbf{1}$ in the solid state supported the involvement of hydrogen bonding in the gelation phenomenon (Figure S4). In general, the gel-based materials displayed $-\mathrm{NH}$ stretch vibration bands at ca. 3311-3257 $\mathrm{cm}^{-1}$, whereas amide $\mathrm{I}(\mathrm{C}=\mathrm{O})$ and amide II vibrations appeared at ca. $1641-1637 \mathrm{~cm}^{-1}$ and $1562-1546 \mathrm{~cm}^{-1}$, which typically correspond to molecules aggregated via hydrogen bonding (non-hydrogen bonded amides display the above vibrations at ca. 3430,1660 and $1515 \mathrm{~cm}^{-1}$ respectively). No vibrational bands were observed in the region $3700-3500 \mathrm{~cm}^{-1}$ (-OH stretching, free) suggesting that the hydroxyl group is also hydrogen bonded, likely to the carbonyl group in an intramolecular manner. Interestingly, the solid and freshly prepared $\mathbf{1}$ showed the selected absorption bands at the same positions within the experimental uncertainty $\left( \pm 2 \mathrm{~cm}^{-1}\right)$, indicating that urea $\mathbf{1}$ is also aggregated via hydrogen bonding in the solid state and hence the existence of some similarity between the solid and the gel structures. In agreement, although compound $\mathbf{1}$ has a very low degree of crystallinity as deduced from its PXRD pattern, the xerogel obtained by freeze-drying the corresponding organogel in toluene still preserved part of this crystallinity (i.e., major broad peak centered at $20^{\circ}, 2(\theta)$ ) (Figures S16-17).

Temperature-dependent ${ }^{1} H$ NMR experiments: As we have observed with peptide-based physical gels, ${ }^{[21]}$ the protons involved in the stabilization of the supramolecular network could be experimentally tracked by NMR experiments at different temperatures. Thus, we recorded ${ }^{1} \mathrm{H}$ NMR spectra of the model organogel made of 1 in $d_{8^{-}}$ toluene within a temperature range where both gel and solution phases could be gradually interconverted. An upfield shift (i.e., $\Delta \delta / \Delta T \approx 5.3 \times 10^{-5} \mathrm{ppm} \mathrm{K}^{-1}$ ) of the $-\mathrm{N} H$ urea protons was first observed in the range $27-35^{\circ} \mathrm{C}$, followed by a clear downfield shift (i.e., $\Delta \delta / \Delta T \approx 1.5 \times 10^{-4} \mathrm{ppm} \mathrm{K}{ }^{-1}$ ) in the range $35-55{ }^{\circ} \mathrm{C}$ (gel phase). Further increase of the temperature until $70{ }^{\circ} \mathrm{C}$ (solution phase) was accompanied by another upfield shift (i.e., $\Delta \delta / \Delta T \approx 3.3$ $\times 10^{-4} \mathrm{ppm} \mathrm{K}^{-1}$ ) (Figure 8).

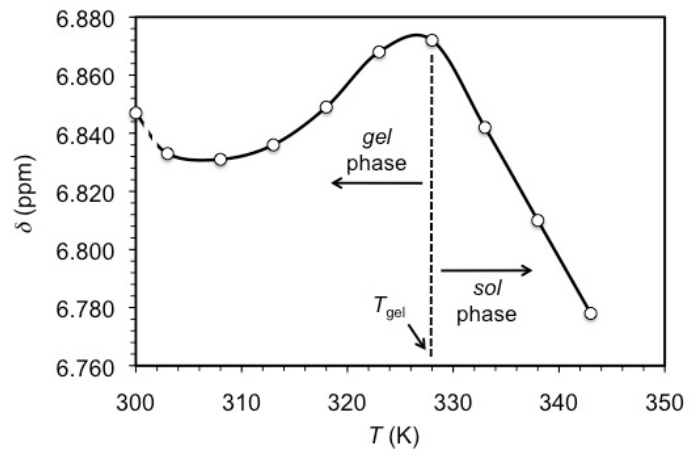

Figure 8. Representative temperature-induced chemical shift of $-\mathrm{N} H$ urea proton of $\mathbf{1}$ in $d_{8}$-toluene.

The unusual and diffident upfield shift observed at the beginning of the experiment is presumably associated to a homogenization process of the sample. The marked inflection point observed at 55 ${ }^{\circ} \mathrm{C}\left(\sim 328 \mathrm{~K}\right.$; breaking of hydrogen bonding) matched with the $T_{\text {gel }}$ of the material. The $-\mathrm{OH}$ proton displayed a very similar chemical shift pattern. All other protons not involved in hydrogen bonding showed the opposite pattern (i.e., upfield shift until $55{ }^{\circ} \mathrm{C}$ and subsequent downfield shift until $70^{\circ} \mathrm{C}$ ) (Figure S6). Overall, these results are in good agreement with the marked influence of hydrogen bonding and $\pi-\pi$ stacking interactions in the gelation process, involving a different type of disassembly processes during the initial heating period. ${ }^{[22]}$

It should be noted that the ${ }^{1} \mathrm{H}$ NMR signals of gelator molecules that form the gel network are unlikely to be observed due to long correlation times. ${ }^{[23]}$ The observed signals are then attributed to small amounts of gelator molecules, either aggregated or disaggregated, dissolved in the immobilized solvent. Thus, the improvement of the signals resolution and increment of their intensity upon heating (Figure S6) is associated to the enhancement of molecular mobility and segregation of the network.

Quantum mechanical calculations: In order to evaluate the strength of intermolecular interactions in 1-7, quantum mechanical calculations at the M06L/6-31+G(d,p) level were performed on model complexes formed by two interacting molecules (dimers). More specifically, seven different complexes were constructed for the dimer of 1 by considering stabilizing $\pi-\pi$ stacking, dispersion, hydrogen bonding and dipole-dipole intermolecular interactions. After this, the seven dimers of $\mathbf{1}$ were used to construct equivalent dimers for 2-7 (i.e. introducing the required changes in the chemical structure without alter the relative orientation between the cores of the two molecules). All these structures were used as starting points for complete geometry optimizations in dichloromethane solution.

Figure 9, which represents the interaction energies calculated in absence of external forces $\left(\Delta \mathrm{E}_{\mathrm{i}}^{\mathrm{gp}}\right)$ and in $\mathrm{CH}_{2} \mathrm{Cl}_{2}$ solution $\left(\Delta \mathrm{E}_{\mathrm{i}}^{\mathrm{sol}}\right)$, indicates that the association is significantly more favored for $\mathbf{1}$ than for the compounds $\mathbf{2 - 7}$, which is fully consistent with the gelation abilities discussed above. Although the interaction energy increases with the polarity of the environment, the functionality and molecular architecture of $\mathbf{1}$ is the most appropriate for the formation of intermolecular interactions. Figure 10A, which depicts the dimer of 1 with lowest $\Delta \mathrm{E}_{\mathrm{i}}^{\mathrm{gp}}$ and $\Delta \mathrm{E}_{\mathrm{i}}^{\mathrm{sol}}\left(-46.4\right.$ and $-27.6 \mathrm{kcal} \mathrm{mol}^{-1}$, respectively) reveals the coexistence of one parallel $\pi-\pi$ stacking, three hydrogen bonds (two $\mathrm{N}-\mathrm{H} \cdots \mathrm{O}$ and one $\mathrm{O}-\mathrm{H} \cdots \mathrm{O}$ ) and one $\mathrm{C}-$ $\mathrm{H} \cdots \pi$ stabilizing interactions. In addition, distances displayed in 
Figure $10 \mathrm{~A}$ are typically associated with strong secondary interactions. Interestingly, the dimer of 7 with lowest $\Delta E_{i}^{g p}$ and $\Delta \mathrm{E}_{\mathrm{i}}^{\mathrm{sol}}$ (-34.6 and $-20.0 \mathrm{kcal} \mathrm{mol}^{-1}$, respectively), which is depicted in Figure 10B, shows the same number and type of interactions. As the only difference between 1 and 7 refers to the $-\mathrm{CF}_{3}$ groups, which have been eliminated in the latter, the reduction in the interaction energies that amounts to ca. $25 \%$ should be attributed to the fluorine-induced electrostatic and dispersive interactions. ${ }^{[24]}$

A)

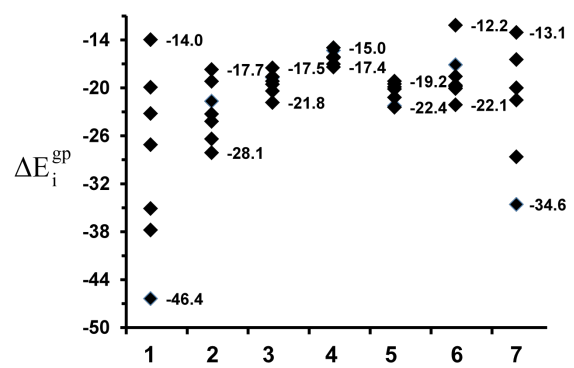

B)

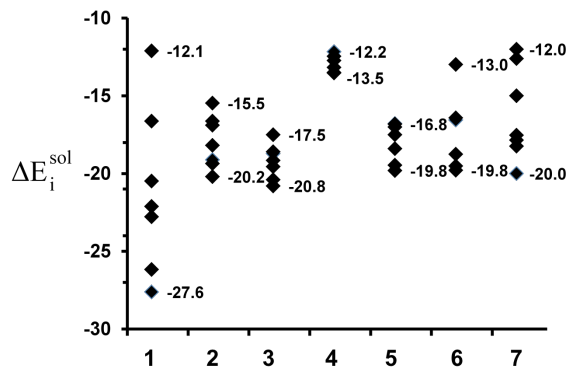

Figure 9. Range of variation of $\mathrm{A}) \Delta \mathrm{E}_{\mathrm{i}}^{\mathrm{gp}}$ and $\left.\mathrm{B}\right) \Delta \mathrm{E}_{\mathrm{i}}^{\text {sol }}$ (both in kcal mol${ }^{-1}$ ) for the calculated dimers of compounds 1-7.

Both the number and, especially, the strength of intermolecular interactions are lower for dimers of compounds $\mathbf{2 - 4}$, as in evidenced in the complex of lowest $\Delta \mathrm{E}_{\mathrm{i}}^{\mathrm{gp}}$ and $\Delta \mathrm{E}_{\mathrm{i}}^{\text {sol }}$ displayed for each compound in Figure S21. This reduction is essentially due to the thiourea in $\mathbf{2}$, which forms weaker hydrogen bonds than the replaced urea, and to the removal of the hydroxyl group in $\mathbf{3}$ and $\mathbf{4}$, which affects not only to intermolecular interactions but also to the interaction of the dimers with the solvent. Thus, the dimeration of 2 results in the formation of two weak $\mathrm{N}-\mathrm{H} \cdots \mathrm{S}$ hydrogen bonds and two parallel $\pi-\pi$ stacking interactions (Figure S21A) while the most stable dimer of $\mathbf{3}$ and $\mathbf{4}$ shows two $\mathrm{N}-\mathrm{H} \cdots \mathrm{O}$ hydrogen bonds and two $\pi-\pi$ stacking interactions, one with the aromatic rings arranged in parallel and the other with a T-shaped disposition (Figures S21B and S21C). Compound 5 deserves special attention since its chemical composition is identical to that of $\mathbf{1}$, the only difference between the two species involving the stereochemistry of the urea group with respect to the five-membered ring. As it can be seen in Figure 10C, which displays the dimer of 5 with lowest $\Delta \mathrm{E}_{\mathrm{i}}^{\mathrm{gp}}$ and $\Delta \mathrm{E}_{\mathrm{i}}^{\mathrm{sol}}\left(-22.4\right.$ and $-20.1 \mathrm{kcal} \mathrm{mol}^{-1}$, respectively), the hydroxyl groups only interact with the solvent, the stereochemistry precluding their participation in stabilizing intermolecular hydrogen bonds. Consequently, the interval of variation of $\Delta \mathrm{E}_{\mathrm{i}}^{\mathrm{gp}}$ and $\Delta \mathrm{E}_{\mathrm{i}}^{\text {sol }}$ is significantly lower for $\mathbf{5}$ than for $\mathbf{1}$. Indeed, comparison of the energies computed for the dimers of the stereoisomers displayed in Figure $10 \mathrm{~A}$ and $10 \mathrm{C}$ indicates that $\mathbf{5}$ is less stable than $\mathbf{1}$ by 8.3 and 11.8 in dichloromethane solution and in the gas-phase, respectively. The most stable dimers of compound 6 in terms of $\Delta \mathrm{E}_{\mathrm{i}}^{\mathrm{gp}}$ and $\Delta \mathrm{E}_{\mathrm{i}}^{\mathrm{sol}}$ are different (Figures S21D and S21E, respectively), even though the characteristics of the secondary interactions found for these complexes are very similar to those described above for $\mathbf{2 - 5}$.
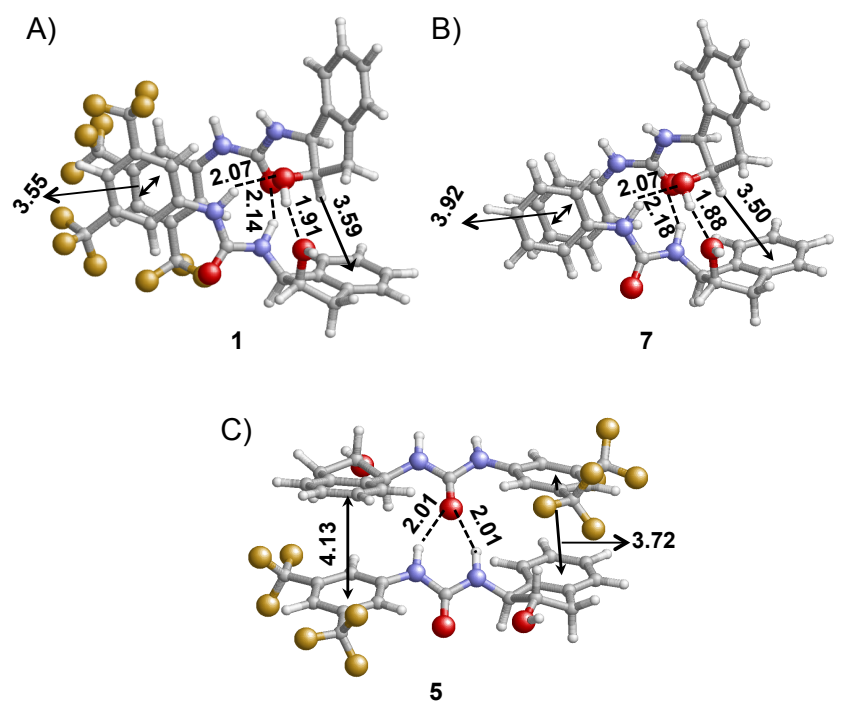

Figure 10. Representation of the most stable complex calculated for dimers of (a) 1, (b) 7 and (c) 5. Intermolecular hydrogen bonds (dashed line), $\pi-\pi$ stacking (double arrow) and $\mathrm{C}-\mathrm{H} \cdots \pi$ interactions (arrow) are displayed. Labels refer to the distances (in $\AA$ ) found for each stabilizing interaction: $\mathrm{H} \cdots \mathrm{O}$ distance in hydrogen bonds; center of masses to center of masses in $\pi-\pi$ stacking; and $\mathrm{H}^{\cdots} \cdots$ center of masses in $\mathrm{C}-\mathrm{H} \cdots \pi$.

Morphological characterization of organogels: The fibrilar nature of the gel networks was evidenced by transmission electron microscopy (TEM) imaging of the corresponding xerogels. Typically, entangled supramolecular fibers with average diameters in the range of 10-30 $\mathrm{nm}$ and a few micrometer lengths were observed for different solvents (Figure 11). Complementary images of dense fibrilar bundles with average heights between $15 \mathrm{~nm}$ and $30 \mathrm{~nm}$ were also obtained by atomic force microscopy (AFM). Interestingly, a close look to the photographs revealed that the fibers corresponding to the gel in some solvents like toluene displayed a helical morphology (Figure 11 and Figure S11).
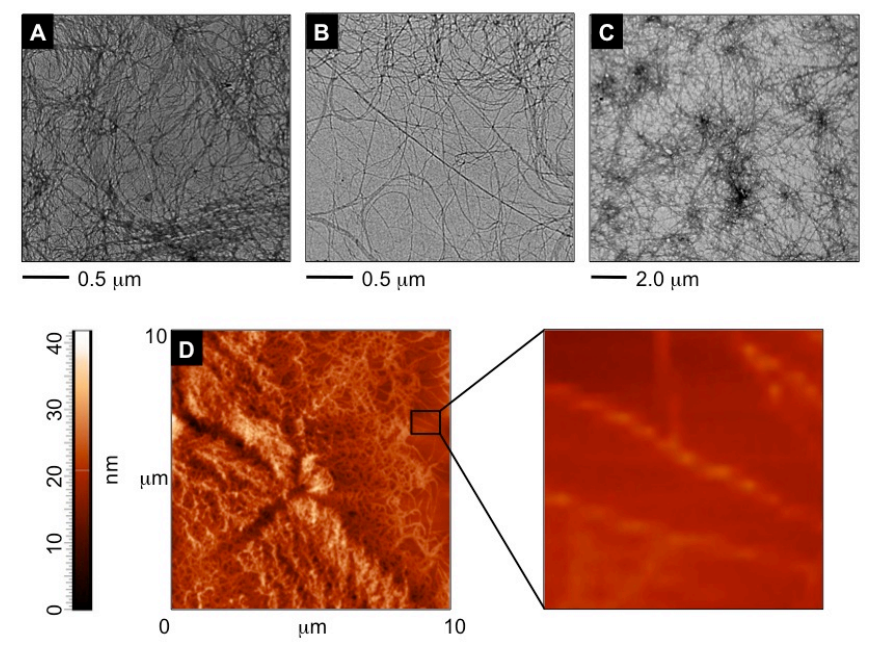

Figure 11. Representative TEM and AFM images of xerogels obtained from the corresponding organogels prepared at the CGC as shown Table 1. The high aspect ratio of the images suggests a highly anisotropic supramolecular assembly. TEM: A-B) toluene; C) methylene chloride. AFM: D) benzene. 
Scanning electron microscopy (SEM) imaging of the xerogels revealed a remarkable influence of the solvent nature on the morphology of the supramolecular aggregates (Figure 12 and Figure S10). For instance, accurate laths of $100-400 \mathrm{~nm}$ widths were obtained with mesitylene (1,3,5-trimethylbenzene), whereas dense ribbon-like fibrilar structures $(\varnothing \sim 10-40 \mathrm{~nm})$ were observed in
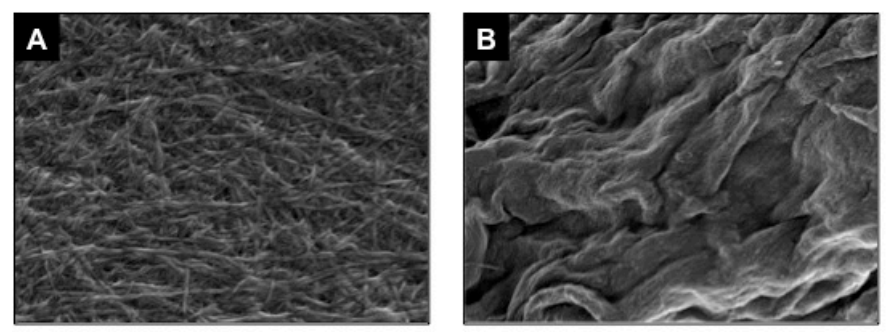

$200 \mathrm{~nm}$
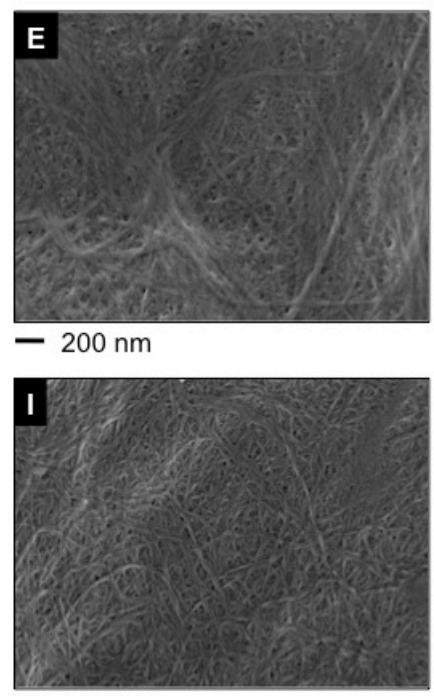

$-200 \mathrm{~nm}$
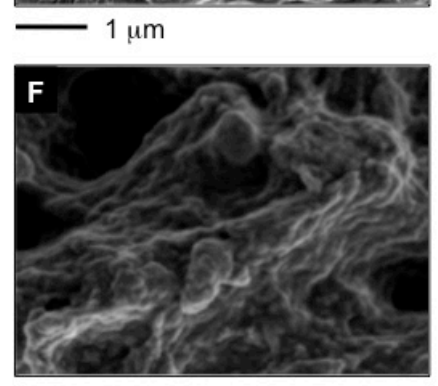

$200 \mathrm{~nm}$

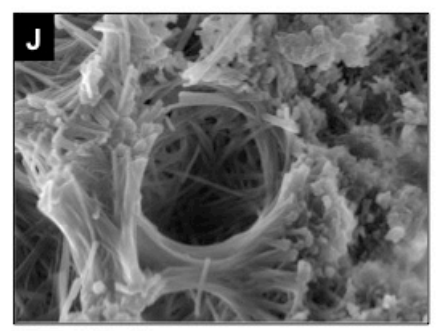

$1 \mu \mathrm{m}$ toluene, benzene and xylenes. In sharp contrast, chlorinated solvents provided fibrilar and highly interconnected macroporous structures ( $\sim 100-500 \mathrm{~nm})$. Other solvents such as nitromethane featured unique lamellar layer microstructures that were not observed with other solvents. The exact mechanism for which each solvent induced a specific morphology remains elusive.

Figure 12. Representative SEM images of xerogels obtained by freeze-drying the corresponding organogels prepared in different solvents at the CGC as shown in Table 1. A) benzene; B) toluene; C) chlorobenzene; D) 1,3-dichlorobenzene; E) 1,2-dichlorobenzene; F) nitrobenzene; G) mesitylene; H) $m$-xylene; I) $o$-xylene; J) methylene chloride; K) carbon tetrachloride; L) nitromethane.

Moreover, the anisotropic and thermoreversible nature of the organogels enabled turn on/off their birefringence under polarized light (Figure 13 and Figure S12), an important property widely searched for optical devices. ${ }^{[25]}$
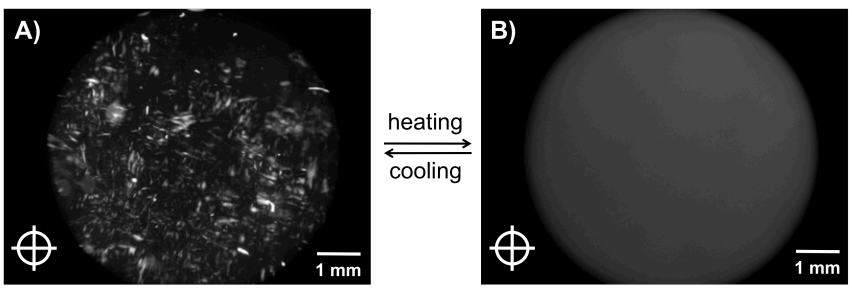

Figure 13. Polarized light microscope images of A) organogel made of $\mathbf{1}$ in methylene chloride at $\mathrm{CGC}$ and $\mathrm{B}$ ) the corresponding solution obtained upon thermal gel-to-sol transition. Polarizing filter is oriented $90^{\circ}$ to the plane of the polarized light.

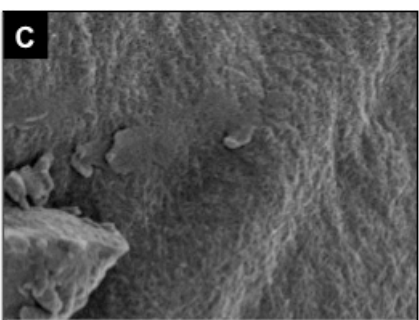

- $100 \mathrm{~nm}$

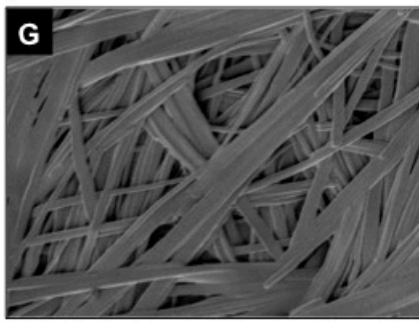

- $1 \mu \mathrm{m}$

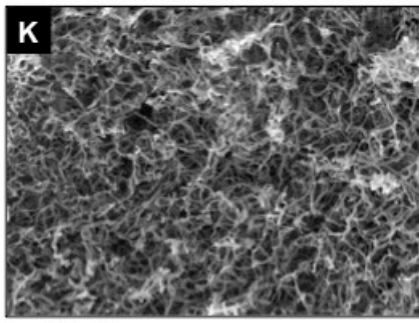

$-1 \mu \mathrm{m}$

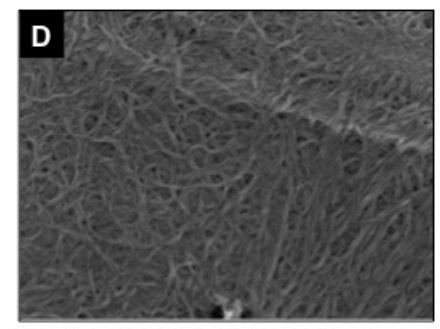

$200 \mathrm{~nm}$

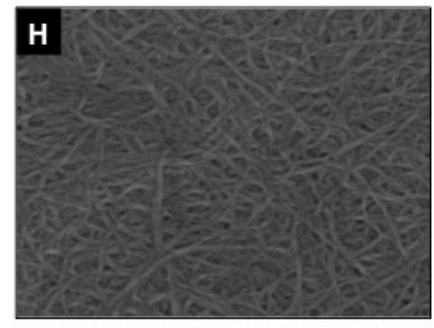

$200 \mathrm{~nm}$

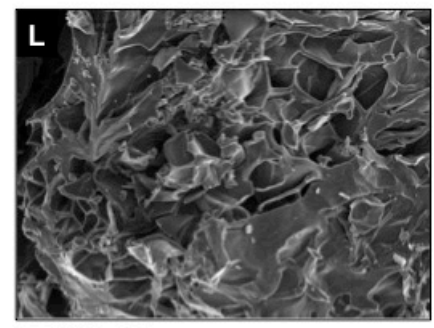

$-10 \mu \mathrm{m}$

Oscillatory rheological measurements: Dynamic rheological measurements of some model materials confirmed their viscoelastic properties. Typically, the storage modulus $G^{\prime}$ and loss modulus $G^{\prime \prime}$ were first measured at room temperature as a function of the frequency (dynamic frequency sweep experiment, DFS) and shear strain (dynamic strain sweep experiment, DSS) to establish the linear viscoelastic regime (Figure 14 and Figures S7-S9). A relatively constant $\tan \delta\left(G^{\prime \prime} / G^{\prime}\right)$ value during the DFS measurement was indicative of a good tolerance of the gel against external forces. Within the linearity limits of deformation (e.g., $1 \mathrm{~Hz}$ frequency and $0.1 \%$ strain), $G^{\prime}$ was found always one order of magnitude higher than $G^{\prime \prime}$ (e.g., $G^{\prime} \approx 2.3 \times 10^{4} \mathrm{~Pa}, G^{\prime \prime} \approx 6.8 \times 10^{3} \mathrm{~Pa}$, for the gel made of 1 in toluene at $5 \mathrm{~g} \mathrm{~L}^{-1}$ ). The stability of the material over time at room temperature was finally confirmed by dynamic time sweep (DTS) measurements at $0.1 \%$ strain and $1 \mathrm{~Hz}$ frequency. 

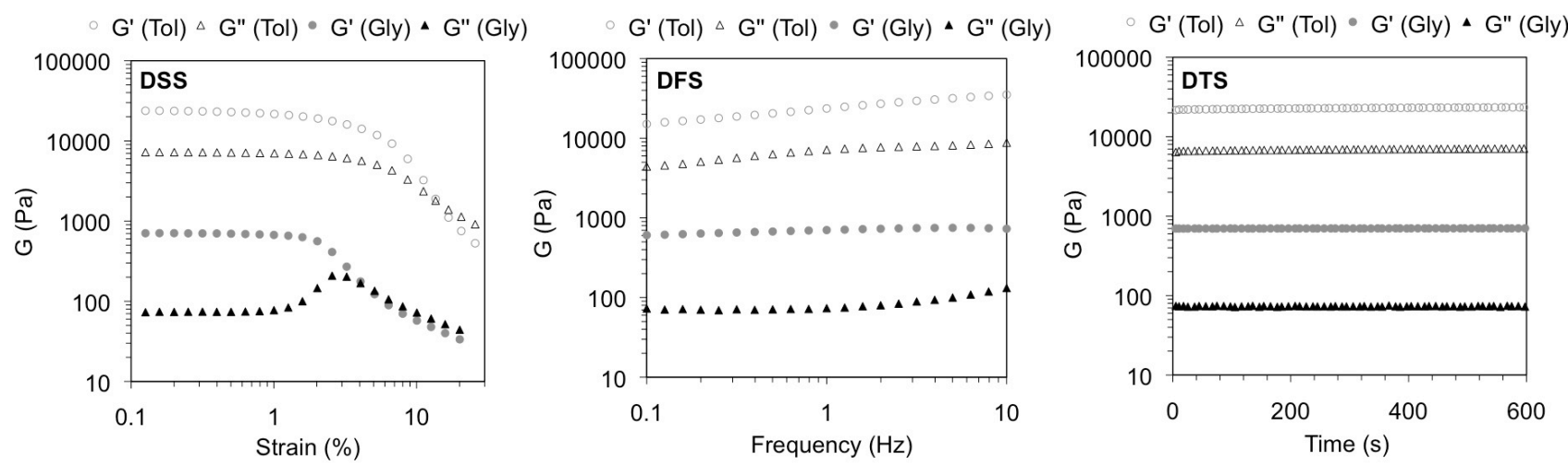

Figure 14. Representative oscillatory rheological experiments (DSS, DFS, DTS) of model gels prepared in toluene and glycerol (90 wt.\%) at $5 \mathrm{~g} \mathrm{~L}{ }^{-1}$.

Interestingly, a thixotropic response ${ }^{[26]}$ to large external strain of the gel made in glycerol was confirmed by a three-steps loop test ${ }^{[27]}$ consisting on the initial application of a shear strain as defined by DTS experiments $\left(G^{\prime}>G^{\prime \prime}\right.$-gel-), further increase of the strain until the gel fractures $\left(G^{\prime}<G^{\prime \prime}-\right.$ sol- $)$ and final return at the same rate to the initial strain value $\left(G^{\prime}>G^{\prime \prime}\right.$-gel-). Figure 15 shows up to $50 \%$ recovery of the original gel strength within $1 \mathrm{~min}$ after the second step and full recovery after $3 \mathrm{~h}$. The thixotropic behavior was also macroscopically observed in a glass vial upon a vigorous shaking/resting cycle. This property is highly pursued for the use of gel-based materials in many real-life applications.

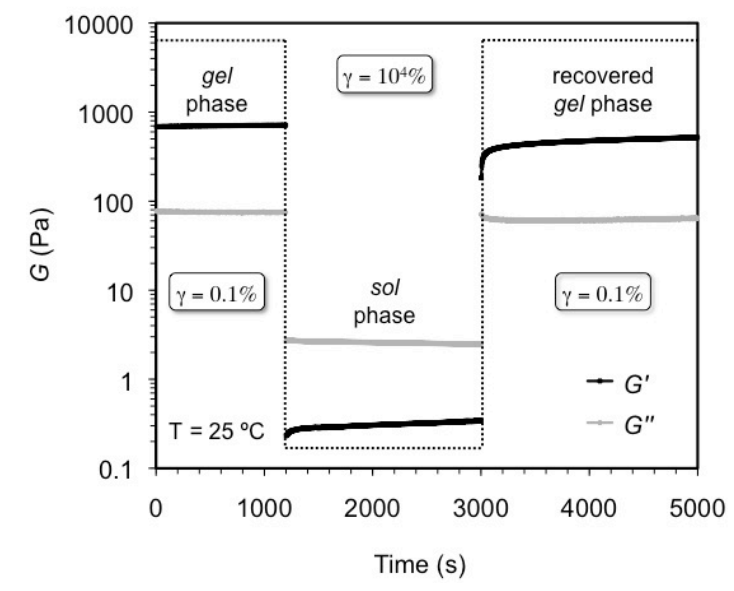

Figure 15. Loop test of the gel made from 1 at CGC in glycerol (90wt.\%). Steps: 1) 1 $\mathrm{Hz}, 0.1 \%$ strain, $20 \mathrm{~min}(\tan \delta=0.1017 \pm 0.002) ; 2) 1 \mathrm{~Hz}, 10000 \%$ strain, $30 \mathrm{~min}$; 3$) 1$ $\mathrm{Hz}, 0.1 \%$ strain $(\tan \delta=0.108 \pm 0.002)$.

Responsiveness to silver ions: Responsiveness tests of model gels in the presence of various ions revealed that they maintained their integrity after incubation with $\mathrm{CuSO}_{4}, \mathrm{NaI}, \mathrm{KOAc}$, or $\mathrm{KNO}_{3}$ aqueous solutions $(0.1 \mathrm{M})$. However, glycerol gel showed an evident color change from colorless to orange-brownish after $30 \mathrm{~min}$ in the presence of solutions containing $\mathrm{Ag}^{+}$ions (e.g., $\mathrm{AgNO}_{3}, \mathrm{AgOAc}$, AgOTf) (Figure 16A). The color change was still visible to the naked eye after $24 \mathrm{~h}$ for concentrations of $\mathrm{Ag}(\mathrm{I})$ ions as low as 0.01 $\mathrm{mM}$. A series of control experiments demonstrated that the counterion was not involved in the alteration of the color. In addition, the presence of the urea was necessary for the optical change, albeit it was neither limited to compound $\mathbf{1}$ nor to the existence of the gel phase. Thus, the color change was also observed either with compound $\mathbf{1}$ at a concentration below the CGC or in the presence of other urea analogous in solution, which is in agreement with the considerable tendency of urea compounds to coordinate $\operatorname{Ag}(\mathrm{I})$ salts. ${ }^{[28]}$ Furthermore, the colorimetric test was also compatible with other solvents (e.g., pinkish and yellowish colors were observed in $\mathrm{CH}_{3} \mathrm{CN}$ and $\mathrm{THF}$, respectively), albeit glycerol provided the best results in terms of color intensity.

In contrast to a solution of urea $\mathbf{1}$, the use of the glycerol gel resulted more convenient as a colorimetric assay. For instance, a piece of gel could be added to the aqueous solution to be tested for the presence of $\operatorname{Ag}(\mathrm{I})$ ions. Afterwards, the gel fragment could be easily separated from the solution by decantation (Figure 16B). UVvis spectroscopy of the colored gel showed a broad absorbance peak in the range 400-500 $\mathrm{nm}$ (Figure S19A), which has been previously associated to the formation of stable silver nanoparticles by simple glycerol oxidation in the absence of any stabilizer. ${ }^{[29]}$
A)

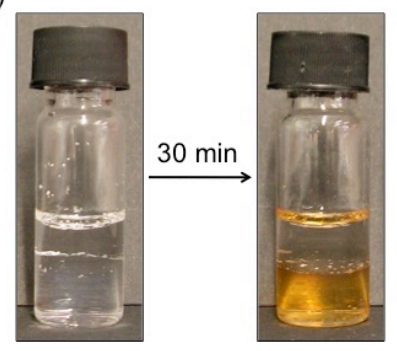

B)

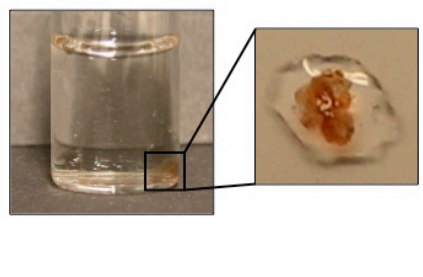

Figure 16. A) Color change of glycerol-gel phase upon addition of a $0.1 \mathrm{M} \mathrm{AgNO}_{3}$ solution on top. B) Detection of $\mathrm{Ag}(\mathrm{I})$ ions in aqueous solution using a piece of glycerol gel made of $\mathbf{1}$ at CGC. Zoom-in: Removal of the piece of gel after coloring.

In situ preparation of gold nanoparticles (Au@NPs): Fine-tuning the dimension of nanoparticles by simple methods is of great interest as the size dispersion is directly related to their electronic structures and chemical reactivities. ${ }^{[30]}$ In this regard, besides the spontaneous silver reduction in the glycerol we explored also the possibility to control the formation of gold nanoparticles by in situ reduction of a suitable precursor. Following the general procedure reported in the literature, ${ }^{[31]}$ gelator 1 was mixed with a toluene solution of $\mathrm{HAuCl}_{4} \cdot 3 \mathrm{H}_{2} \mathrm{O}(5 \mathrm{mM})$ containing a phase transfer agent such as tetraoctylammonium bromide (TOAB). A yellowish gel containing $\mathrm{Au}(\mathrm{III})$ ions was then obtained by the heating-cooling method. The addition of a reducing solution of tetraoctylammonium borohydride on top of the gel caused the slow formation of an intense purple- 
colored layer. Different concentrations of gold precursor and reducing agents were investigated but in all cases the gel structure turned finally into a stable dark purple solution. Nevertheless, the same experiment was carried out in solution in the absence of gelator 1 for comparative purposes. In this case, the color change took place immediately upon addition of the reducing agent. The surface plasmon resonance band centered around $550 \mathrm{~nm}$ (Figure S19B) and comparative TEM imaging of the purple materials proved the successful formation of nearly spherical gold nanoparticles (Figure 17). Very interestingly, although we could not achieve the formation of stable gel-nanoparticle hybrid materials, the gel network made of $\mathbf{1}$ behaved as a nanoreactor slowing down the kinetics of the reduction to form smaller and better-dispersed nanoparticles (average particle size ca. $15 \pm 5 \mathrm{~nm}$ ) in comparison to those obtained in solution (average particle size ca. $45 \pm 10 \mathrm{~nm}$ ).
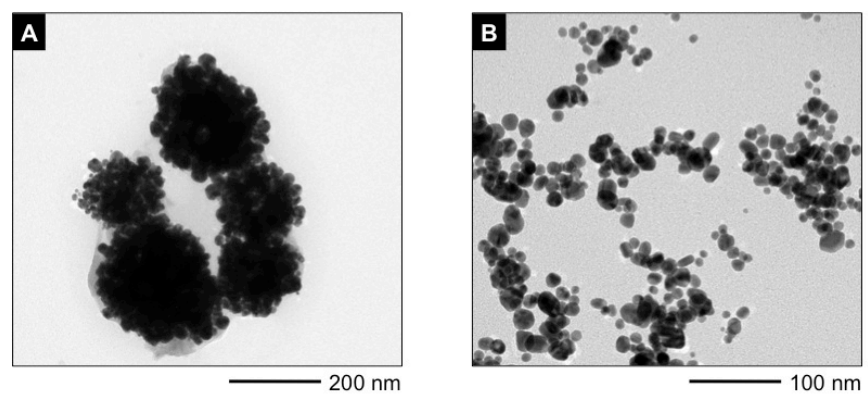

Figure 17. TEM images of the gold nanoparticles obtained from the precursor seeded A) in toluene solution and B) in toluene gel prepared at CGC.

Catalytic alkylation reaction in gel media: During the last decade, a number of publications have shown the potentiality of functional gels as recyclable catalysts and/or reaction vessels with enhanced selectivity. ${ }^{[32]}$ Within our research program devoted to investigate reactivities in organized and confined media, we also decided to examine the ability of the self-assembled gel network made from 1 to serve as a nanoreactor for the metal-free Friedel-Crafts alkylation of $1 \mathrm{H}$-indole with trans- $\beta$-nitrostyrene (Scheme 2). We have previously studied both urea $\mathbf{1}$ and thiourea $\mathbf{2}$ as organocatalysts for this reaction in solution. ${ }^{[9]}$ The results obtained there clearly demonstrated a higher catalytic efficiency for the thiourea, which was attributed to its greater hydrogen bond donor ability and less tendency to self-assembly in comparison to $\mathbf{1}$. Remarkably, when the above reaction was carried out in the gel phase provided by $\mathbf{1}$ in toluene, the product $\mathbf{8}$ yield increased ca. 1.4-fold compared with that in solution (Figure S18).

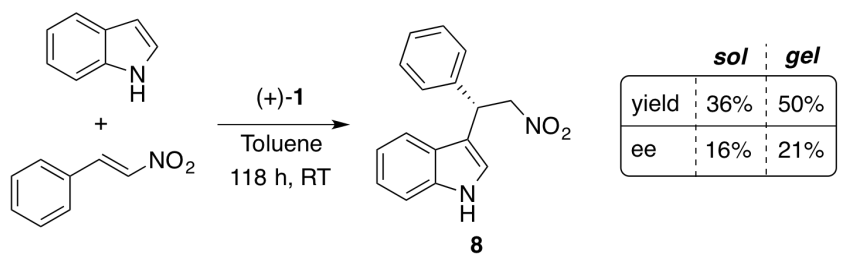

Scheme 2. Alkylation of $1 H$-indole with trans- $\beta$-nitrostyrene in solution and gel phase.

This result is especially relevant if we consider that kinetics of diffusion-controlled processes can be 10-20 times faster in stirred solutions than in non-stirred gel media. ${ }^{[33]}$ In addition, the average level of enantioselectivity observed in gel phase was slightly superior than in solution, suggesting that the fibrilar network could somehow provide an additional shielding effect responsible for facial discrimination. In addition, the supramolecular porous network could also contribute to some level of catalyst spatial isolation, which has been elegantly achieved by means of porous MOF environments where the reaction occurs primarily within the pores. $^{[34]}$

\section{Conclusion}

The results of this study confirm the potential of some urea-based organocatalysts such as $\mathbf{1}$ as building block for the preparation of physical organogels at concentrations ranging from 3 to $50 \mathrm{~g} \mathrm{~L}^{-1}$. According to FT-IR, NMR and quantum mechanical studies, the major driving forces for the gelation of organic solvents by $\mathbf{1}$ are hydrogen bonding and $\pi-\pi$ interactions. In comparison to the Hansen solubility parameters, the Kamlet-Taft solvatochromic parameters offer here a more convenient scenario to rationalize the gelation ability. Moreover, a variety of morphologies including helical, laths, macroporous or lamellar nanostructures could be obtained depending on the solvent nature. Variations of the most important structural segments (compounds 2-7) that could influence the self-assembly of $\mathbf{1}$ and computer modeling proved the existence of unique molecular interactions in this molecule that drive the formation of stable hierarchical supramolecular aggregates.

Multistimuli responsive behaviors (e.g., thermal, mechanical, optical and chemical responses) and a multifunctional nature were demonstrated in some of the gel materials. In this respect, $\mathbf{1}$ could be used for the phase selective gelation of oil/water mixtures, the gel in glycerol was found to be thixotropic and provided a sensing ability for $\mathrm{Ag}(\mathrm{I})$ ions at millimolar concentrations in aqueous solutions. In addition, the gel matrix obtained in toluene behaved as a nanoreactor for the in situ formation of dispersed gold nanoparticles with average size of $15 \mathrm{~nm}$, and for the Friedel-Crafts alkylation of $1 H$-indole with trans- $\beta$-nitrostyrene catalyzed by $\mathbf{1}$. Efforts towards the development of other multifunctional materials are currently underway in our laboratories.

\section{Experimental section}

A) Synthesis and characterization of compounds.

Materials. All commercially available solvents and reagents for synthesis and analysis were used as received without further purification. Compound $\mathbf{4}$ was available from commercial sources.

Characterization methods. Purification of reaction products was carried out by flash chromatography using silica-gel $(0.063-0.200 \mathrm{~mm})$ or medium pressure liquid chromatography using prepacked silica columns. Analytical thin layer chromatography (TLC) was performed on $0.25 \mathrm{~mm}$ silica gel $60-\mathrm{F}$ plates. The products were visualized by exposure to UV light $(254 \mathrm{~nm})$ and phosphomolybdic acid as stain. Mass spectra were obtained using ESI ionization on a Bruker Daltonics Esquire 3000 plus (MicroTofQ) spectrometer. Unless otherwise indicated, NMR spectra were recorded at room temperature on a Bruker AVANCE-II instrument. ${ }^{1} \mathrm{H}$ NMR spectra were recorded at $400 \mathrm{MHz}$ and ${ }^{13} \mathrm{C}$ NMR spectra were recorded at $100 \mathrm{MHz}$, using DMSO- $d_{6}$ and $\mathrm{D}_{3} \mathrm{CCOCD}_{3}$ as the deuterated solvents. Chemical shifts were reported in the $\delta$ scale relative to residual DMSO (2.50 ppm for ${ }^{1} \mathrm{H}$ NMR and $39.43 \mathrm{ppm}$ for $\left.{ }^{13} \mathrm{C} \mathrm{NMR}\right)$ and acetone (2.05 ppm for ${ }^{1} \mathrm{H}$ NMR). Coupling constants $(J)$ were expressed in Hertz. Melting points were determined on a Gallenkamp variable heating apparatus. Optical rotations were measured in a JASCO DIP-370 polarimeter. IR spectra were recorded on 
a Nicolet Avatar 360 FT-IR spectrophotometer. High performance liquid chromatography (HPLC) was carried out using a Waters 2695 Alliance detector.

General procedure for the synthesis of compounds 1-3, 5-7. To a stirred solution of 3,5-bis(trifluoromethyl)phenyl isocyanate $(1.1 \mathrm{mmol}$ ) (for the synthesis of compounds 1 , 3, 5 and 6), 3,5-bis(trifluoromethyl)phenyl isothiocyanate (1.1 mmol) (for the synthesis of compound 2) or phenyl isocyanate $(1.1 \mathrm{mmol}$ ) (for the synthesis of compound 7 ) in $\mathrm{CH}_{2} \mathrm{Cl}_{2}(5 \mathrm{~mL})$, the corresponding commercially available amine $(1.0 \mathrm{mmol})$ (i.e., $(1 S, 2 R)$-1-amino-2,3-dihydro-1 $H$-inden-2-ol for compounds $\mathbf{1}, 2$ and 7 ; $(1 R, 2 R)-1$ amino-2,3-dihydro- $1 H$-inden-2-ol for compound 5; 3,5-bis(trifluoromethyl)aniline for compound 3; $(R)$-2,3-dihydro-1 $H$-inden-1-amine for compound $\mathbf{6}$ ) was added in one portion. After stirring the resulting solution at room temperature overnight, the solvent was evaporated under reduced pressure and the product was purified by flash chromatography or medium pressure liquid chromatography $\left(\mathrm{SiO}_{2}\right.$, hexane/EtOAc 7:3). ${ }^{1} \mathrm{H}$ and ${ }^{13} \mathrm{C}$ NMR spectra for compounds (+)-1, ${ }^{[35]}(-)-\mathbf{1},{ }^{[36]} \mathbf{2},{ }^{[9]}$ and $\mathbf{3}^{[37]}$ were consistent with values previously reported in the literature.

\section{1-(3,5-Bis(trifluoromethyl)phenyl)-3-((1R,2R)-2-hydroxy-2,3-dihydro-1H-inden-1-}

yl)urea (5): Following the general procedure, compound 5 was obtained as white solid in $92 \%$ yield: M.p. $232-234{ }^{\circ} \mathrm{C}$. $[\alpha]^{20}{ }_{\mathrm{D}}-88.1$ (c 0.74, DMSO). ${ }^{1} \mathrm{H}$ NMR $(400 \mathrm{MHz}$, DMSO-d $\left.d_{6}\right) \delta 9.21($ br s, $1 \mathrm{H}), 8.14(\mathrm{~s}, 2 \mathrm{H}), 7.57(\mathrm{~s}, 1 \mathrm{H}), 7.22-7.19(\mathrm{~m}, 4 \mathrm{H}), 6.89$ (d, $J=$ $8.3 \mathrm{~Hz}, 1 \mathrm{H}), 5.33(\mathrm{~d}, J=5.5, \mathrm{~Hz}, 1 \mathrm{H}), 4.95(\mathrm{dd}, J=6.7,8.1 \mathrm{~Hz}, 1 \mathrm{H}), 4.29-4.22(\mathrm{~m}, 1 \mathrm{H})$, 3.14 (dd, $J=7.1,15.6 \mathrm{~Hz}, 1 \mathrm{H}), 2.72(\mathrm{dd}, J=7.27,15.6 \mathrm{~Hz}, 1 \mathrm{H}) .{ }^{13} \mathrm{C} \mathrm{NMR}(100 \mathrm{MHz}$, DMSO- $\left.d_{6}\right) \delta 154.9,142.4141 .8,139.6,130.5\left(\mathrm{q}, J=32.5 \mathrm{~Hz}, C_{C F}\right), 127.6,126.6$, 124.6, 123.8, 123.3 (q, $\left.J=272.8 \mathrm{~Hz}, C_{3}\right), 117.5-117.2(\mathrm{~m}, 1 \mathrm{C}), 113.6-113.4(\mathrm{~m}, 1 \mathrm{C})$, 77.9, 61.6, 38.5. IR (KBr film) $\left(\mathrm{cm}^{-1}\right) \vee$ 3395, 3326, 2923, 2853, 1634, 1278, 1129, 1073, 749. MS (ESI) calcd for $\mathrm{C}_{18} \mathrm{H}_{14} \mathrm{~F}_{6} \mathrm{~N}_{2} \mathrm{NaO}_{2} 427.1$; found $427.1[\mathrm{M}+\mathrm{Na}]$.

\section{(R)-1-(3,5-Bis(trifluoromethyl)phenyl)-3-(2,3-dihydro-1H-inden-1-yl)urea}

(6):

Following the general procedure, compound 6 was obtained as white solid in $75 \%$ yield. M.p. $215-217^{\circ} \mathrm{C}$. $[\alpha]^{22}{ }_{\mathrm{D}}-48.7$ (c 0.77, DMSO). ${ }^{1} \mathrm{H}$ NMR (400 MHz, $\left.\mathrm{CD}_{3} \mathrm{COCD}_{3}\right) \delta 8.58$ (br s, 1H), $8.20(\mathrm{~s}, 2 \mathrm{H}), 7.55(\mathrm{~s}, 1 \mathrm{H}), 7.36-7.34(\mathrm{~m}, 1 \mathrm{H}), 7.26-7.17(\mathrm{~m}, 3 \mathrm{H}), 6.34$ (br d, $J$ $=7.3 \mathrm{~Hz}, 1 \mathrm{H}), 5.35(\mathrm{q}, J=7.7 \mathrm{~Hz}, 1 \mathrm{H}), 2.96(\mathrm{ddd}, J=3.6,8.7,15.9 \mathrm{~Hz}, 1 \mathrm{H}), 2.89-2.81$ $(\mathrm{m}, 1 \mathrm{H}), 2.59-2.51(\mathrm{~m}, 1 \mathrm{H}), 1.91-1.82(\mathrm{~m}, 1 \mathrm{H}) .{ }^{13} \mathrm{C}$ NMR $\left(100 \mathrm{MHz}\right.$, DMSO- $\left.d_{6}\right) \delta$ 154.6, 143.9, 142.7, 142.4, 130.5 (q, $J=32.5 \mathrm{~Hz}, C \mathrm{CF}_{3}$ ), 127.4, 126.3, 124.5, 123.7, $123.3\left(\mathrm{q}, J=272.8 \mathrm{~Hz}, C \mathrm{~F}_{3}\right), 117.4-117.3(\mathrm{~m}, 1 \mathrm{C}), 113.6-113.4(\mathrm{~m}, 1 \mathrm{C}), 54.5,33.3$, 29.5. IR (KBr film) $\left(\mathrm{cm}^{-1}\right) \vee 2923,2853,1639,1457,1276,1130$. MS (ESI) calcd for $\mathrm{C}_{18} \mathrm{H}_{14} \mathrm{~F}_{6} \mathrm{~N}_{2} \mathrm{NaO} 411.1$; found $411.1[\mathrm{M}+\mathrm{Na}]$.

1-((1S,2R)-2-Hydroxy-2,3-dihydro-1H-inden-1-yl)-3-phenylurea (7): Following the general procedure, compound 7 was obtained as white solid in 94\% yield. M.p. 224-226 ${ }^{\circ} \mathrm{C} .[\alpha]^{21}{ }_{\mathrm{D}}+53.9$ (c 0.64, DMSO). ${ }^{1} \mathrm{H}$ NMR (400 MHz, DMSO- $\left.d_{6}\right) \delta 8.85$ (s, 1H), 7.45 (d, $J=8.5 \mathrm{~Hz}, 1 \mathrm{H}), 7.26-7.18(\mathrm{~m}, 6 \mathrm{H}), 6.91(\mathrm{t}, J=7.3 \mathrm{~Hz}, 1 \mathrm{H}), 6.44(\mathrm{~d}, J=8.7 \mathrm{~Hz}, 1 \mathrm{H})$, $5.24(\mathrm{~d}, J=4.2 \mathrm{~Hz}, 1 \mathrm{H}), 5.10(\mathrm{dd}, J=5.0,8.5 \mathrm{~Hz}, 1 \mathrm{H}), 4.45(\mathrm{dd}, J=4.1,8.9 \mathrm{~Hz}, 1 \mathrm{H})$, 3.07 (dd, $J=4.8,16.2 \mathrm{~Hz}, 1 \mathrm{H}), 2.80(\mathrm{~d}, J=16.2 \mathrm{~Hz}, 1 \mathrm{H}) .{ }^{13} \mathrm{C}$ NMR $(100 \mathrm{MHz}$, DMSO$\left.d_{6}\right) \delta 155.2,143.1,140.5,140.3,128.6,127.0,126.2,124.8,123.8,120.9,117.4,72.0$, 57.1, 39.6. IR (KBr film) $\left(\mathrm{cm}^{-1}\right) \vee$ 3469, 3365, 3292, 2923, 2854, 1623, 1568, 1458, 1246, 1050, 766, 748, 735. MS (ESI) calcd for $\mathrm{C}_{16} \mathrm{H}_{16} \mathrm{~N}_{2} \mathrm{NaO}_{2} 291.1$; found 291.1 $[\mathrm{M}+\mathrm{Na}]$.

\section{B) Preparation and characterization of gel materials.}

Characterization methods. Oscillatory rheological measurements were performed at 25 ${ }^{\circ} \mathrm{C}$ with an AR 2000 Advanced Rheometer from TA Instruments equipped with a cooling system (Julabo C). A $20 \mathrm{~mm}$ plain plate geometry (stainless steel) was used. Dynamic strain sweep (DSS) measurements were first carried out between $0.01 \%$ and $100 \%$ strain at $1 \mathrm{~Hz}$ frequency to estimate the strain value at which reasonable torque values were given (i.e., about 10 times of the transducer resolution limit). Dynamic frequency sweep (DFS) measurements (i.e., from 0.1 to $10 \mathrm{~Hz}$ at $0.1 \%$ strain) and time sweep measurements (DTS) within the viscoelastic regime (i.e., $0.1 \%$ strain, $1 \mathrm{~Hz}$ frequency) were subsequently performed. Additionally, the thixotropic behavior of the gels was investigated by a 3-steps loop experiment: (1) Application of a low shear strain as established by previous DTS experiments (the material is in the gel state, $G^{\prime}>G^{\prime \prime}$ ), (2) increase of the shear strain until the gel fractures (the material turns into a viscous solution, $G^{\prime}<G^{\prime \prime}$ ), and (3) return at the same rate to the initial strain \% value (the gel phase has been recovered, $G^{\prime}>G^{\prime \prime}$ ). FT-IR spectra were recorded using a Diamond ATH (attenuated total reflection) accessory (Golden Gate) in a VARIAN 1000 FT-IR Scimitar $^{\mathrm{TM}}$ Series) spectrophotometer. Morphological characterization of the samples was carried out by transmission electron microscopy (TEM), field-emission scanning electron microscopy (FESEM) and atomic force microscopy (AFM). a) TEM: Images were recorded using a JEOL-2000 FXII transmission electron microscope (resolution = $0.28 \mathrm{~nm}$ ) equipped with a CCD Gatan 694 digital camera and operating at $10 \mathrm{kV}$ (accelerating voltage). Sample preparation: $10 \mu \mathrm{L}$ of the gel suspension was allowed to adsorb for $30 \mathrm{~s}$ onto carbon-coated grids ( $300 \mathrm{mesh}$, from TED PELLA, Inc.). After the adsorption, the excess solvent was removed by touching the edges with a small piece of filter paper. The specimens were then dried overnight in a desiccator at low pressure and RT. b) FESEM: Images were obtained with a Carl Zeiss Merlin field emission scanning electron microscope (FESEM, resolution $=0.8 \mathrm{~mm}$ resolution) equipped with a digital camera and operating at $10 \mathrm{kV}$ (accelerating voltage) and $10 \mathrm{~mA}$ (emission current). Sample preparation: Specimens were prepared by the freeze-drying method. ${ }^{[38]}$ Prior to imaging, a $5 \mathrm{~nm}$ sized Pt film was sputtered (40 mA, 30 seconds) on the samples placed on carbon tape. c) AFM: Imaging was performed on a Ntegra Aura (NTMDT) instrument in tapping mode at $1 \mathrm{~Hz}$ scanning rate using directly polycrystalline sapphire $(24 \times 19.3 \times 0.5 \mathrm{~mm})$ as substrate and a single crystal silicon tip coated with TiN (NSG01/TiN, 0.01-0.025 $\Omega$-cm, Antimony doped) at 200-400 kHz drive frequency. Drive amplitude ranged from 60 to $100 \mathrm{mV}$. Sample preparation: 5-10 $\mu \mathrm{L}$ of a gel suspension (ca. 10-fold dilution in the corresponding solvent) was placed on the substrate and homogeneously dispersed with a spatula to form a thin layer that was allowed to dry in air for at least 30-60 min before measurement. The growth of crystals in gel phases was monitored using a Wild Makroskop M420 optical microscope equipped with a Canon Power shot A640 digital camera for digital imaging. An additional polarization filter was used to observe the gel material under polarized light. Differential scanning calorimetry (DSC) spectra were measured on a DSC7 (Perkin Elmer) instrument at a scan rate of $10{ }^{\circ} \mathrm{C} \min ^{-1}$ under nitrogen atmosphere. For the measurements, an appropriate amount of gel was placed into a pre-weighted $\mathrm{Al}$ pan, which was sealed and weight on a six-decimal plate balance. The pans were weighted again after each measurement to check for possible leakage. Temperature-dependent ${ }^{1} \mathrm{H}$ NMR studies were carried out on a $400 \mathrm{MHz}$ Bruker Avance instrument equipped with a BVT 2000 heating system (Bruker BioSpin $\mathrm{GmbH}$ ). Specific surface area, pore volume, pore size and gas adsorption/desorption isotherms were measured by a Micromeritics ASAP 2020 analyzer at $77 \mathrm{~K}$ after vacuum degassing of the sample at 80 ${ }^{\circ} \mathrm{C}$ for $24 \mathrm{~h}$. Powder X-ray diffraction (PXRD) patterns were collected on a Rigaku D/max-2500 rotating-anode powder diffractometer with $\mathrm{CuK} \alpha$ radiation operated at 40 $\mathrm{kV}$ and $80 \mathrm{~mA}$. Conditions: $0.03^{\circ}$, time $5 \mathrm{~s} / \mathrm{step}, 2$ theta range $5-60^{\circ}$. Distilled water contact angles and surface energies were measured with a PG goniometer (ASTM D5946) with the droplet size $(4 \mu \mathrm{L})$ controlled by a pump-dosing unit. Absorption spectra were recorded on a Varian Cary BIO 50 UV-vis scanning spectrophotometer using $1 \mathrm{~cm}$ quartz cuvettes (Suprasil ${ }^{\circledR}$, Hellma). Minimum gelation concentrations, MGC, were estimated by adding solvent in several portions $(0.1 \mathrm{~mL}$ each $)$ into the vial where no gelation was achieved at the previous concentration and some material remained insoluble. The initial concentration for gelation tests was $5 \mathrm{~g} \mathrm{~L}^{-1}$. The state of the mixture was determined after the heating-cooling cycle as described above. New tests were carried out at lower concentration if stable clear solutions were obtained at 5 $\mathrm{g} \mathrm{L}^{-1}$. Gel-to-sol transition temperatures, $T_{\text {gel }}$, were typically determined by the inverse flow method. The average values of at least two random experiments were given. The seal vial containing the organogel was hung horizontally into an oil bath, which was heated up at $2{ }^{\circ} \mathrm{C} \min ^{-1}$. Herein, the temperature at which the gel started to break was defined as $T_{\text {gel. }}$. These values were correlated with the first DSC endothermic transition of selected examples. 
General procedure for the preparation of organogels. Solvents used for gelation tests were purchased from commercial suppliers and were at least of p.a. quality. Typically, a weighted amount of the corresponding compound (1-7) and $1 \mathrm{~mL}$ of the appropriate solvent were placed into a screw-capped glass vial $(4.5 \mathrm{~cm}$ length $\times 1.2 \mathrm{~cm}$ diameter). The closed vial was gently heated with a heat gun until the solid material was completely dissolved. The resulting isotropic solution was then spontaneously cooled down to room temperature. The material was preliminary classified as gel if it did not exhibit gravitational flow upon turning the vial upside-down at room temperature. The gel state was further confirmed by rheological measurements.

Phase selective gelation tests: In a screw-capped glass vial $(4.5 \mathrm{~cm}$ length $\times 1.2 \mathrm{~cm}$ diameter) were subsequently added the amount of the gelator (+)-1 according to its MGC, $1.0 \mathrm{~mL}$ of the desired organic solvent and $1.0 \mathrm{~mL}$ of distilled water. The vial was closed and gently heated with a heat gun until the gelator was dissolved. The state of the material was evaluated after cooling down the mixture to room temperature by turning the vial upside-down.

Responsiveness experiments: $1.0 \mathrm{~mL}$ of gel material was prepared at the corresponding MGC as described above. The gel was allowed to equilibrate for at least $1 \mathrm{~h}$ before 1.0 $\mathrm{mL}$ of test solution (e.g., $1.0 \mathrm{~mL}$ with $0.1 \mathrm{M} \mathrm{AgNO}_{3}$ ) was placed on top of the gel. The effect of the test solution on the gel (e.g., induction to gel-to-sol transition, color change) was monitored over time at room temperature.

Synthesis of gold nanoparticles (Au@NPs): Au@NPs were prepared following the general procedure previously described in the literature. ${ }^{[31]}$ Typically, an aqueous solution of $\mathrm{HAuCl}_{4} \cdot 3 \mathrm{H}_{2} \mathrm{O}$ ( $5 \mathrm{~mL}$ from a $5 \mathrm{mM}$ stock solution) was mixed with toluene $(5 \mathrm{~mL})$ in the presence of equimolar phase transfer catalyst TOAB. A gel was subsequently prepared by the heating-cooling method using $3.0 \mathrm{mg}$ of the urea gelator (+)-1 in $1.0 \mathrm{~mL}$ of the $\mathrm{Au}(\mathrm{III})$-containing toluene solution. A stock solution of tetraoctylammonium borohydride was prepared in situ by stirring $\mathrm{NaBH}_{4}(5 \mathrm{mg}, 0.11$ $\mathrm{mmol})$ and TOAB $(2 \mathrm{mg}, 0.004 \mathrm{mmol})$ in toluene $(4 \mathrm{~mL}) .200 \mu \mathrm{L}$ of this solution was added on top of the toluene gel to promote the formation of the nanoparticles. The formation of Au@NPs was confirmed by TEM and UV-vis spectroscopy. The specimens for TEM were prepared by diluting $20 \mu \mathrm{L}$ of the formed purple phase with $20 \mu \mathrm{L}$ of toluene. A drop of this solution was placed on the carbon-coated copper grid and allowed to dry before the measurements.

Friedel-Crafts alkylation in gel media: Dry toluene $(0.1 \mathrm{~mL})$ was added to screwcapped vial containing a mixture of trans- $\beta$-nitrostyrene $(0.1 \mathrm{mmol}), 1 H$-indole $(0.15$ $\mathrm{mmol})$ and $20 \mathrm{~mol} \%$ of the urea gelator $(+)-1$. The mixture was gently heated until an isotropic solution was formed. Complete gelation occurred within $20 \mathrm{~min}$ and the reaction was allowed to proceed for $118 \mathrm{~h}$ at room temperature. After this time, the solvent was removed under reduced pressure. The crude product was analyzed by NMR using DMA (0.1 mol) as internal standard. For HPLC analysis, the product was purified by column chromatography using $n$-hexanes/ethyl acetate $8: 2$ as eluent.

C) Quantum mechanical calculations. Quantum mechanical calculations were performed using the Gaussian $09^{[39]}$ computer program, applying default therholds and algorithms. The meta-generalized gradient approximation (GGA) functional M06L of Truhlar and $\mathrm{Zhao}^{[40]}$ was combined with the $6-31+\mathrm{G}(\mathrm{d}, \mathrm{p})^{[41]}$ basis set for calculations on dimers of 1-7. The M06L function is known to provide geometry and interaction energy of dimers stabilized by non-covalent interactions, including $\pi$-stacking, with accuracy close to that of coupled cluster with both single and double substitutions (CCSD). ${ }^{[22]}$ Environmental effects (here dichloromethane) have been accounted for using the wellknown Polarizable Continuum Model (PCM) model. ${ }^{[43]}$ Complete geometry optimizations of all dimer were performed using the PCM approach. Intermolecular interaction energies in the gas phase (i.e., in absence of environmental forces) were estimated as the difference between the energy in the gas-phase of the dimer optimized in dichloromethane solution and the energies of the isolated subsystems in the gas-phase with the geometries obtained from the optimization in solution of the dimer. The basis set superposition error of the energies of the subsystems were corrected using the counterpoise (CP) method. ${ }^{[44]}$ Similarly, intermolecular interaction energies in dichloromethane were calculated as the difference between the energies in solution of the dimer and the subsystems.

\section{Acknowledgements}

Financial support from Universität Regensburg (Förderlinie C des Finanziellen Anreizsystems für Drittmitteleinwerbung), Spanish Ministry of Economía y Competitividad (MINECO, Madrid, Spain, Projects CTQ2010-19606 and MAT201234498), and the Governments of Aragón (Zaragoza, Spain, Research Group E-10) and Catalunya (research group 2009 SGR 925, XRQTC and "ICREA Academia" Award for excellence in research to C.A.) is gratefully acknowledged. We thank Prof. Achim Göpferich (Universität Regensburg) for granting us access to the AR 2000 rheometer, Prof. Pilar Cea, Dr. Santiago M. Solans, and Dr. Reyes Mallada (Universidad de Zaragoza), Dr. Rahul Banerjee and Mr. Pradip Pachfule (NCL, Pune, India), for discussions and/or helpful assistance with some measurements. C.A. is indebted to the Centre de Supercomputació de Catalunya (CESCA) for the computational resources provided. D.D.D. thanks the DFG for the Heisenberg Professorship Award.

[1] S. M. Mukhopadhyay, Nanoscale Multifunctional Materials: Science and Applications, John Wiley \& Sons, Inc., Hoboken, NJ, US, 2011.

[2] a) X. Y. Liu, Top Curr. Chem. 2005, 256, 1-37; b) M. de Loos, B. L. Feringa, J. H. van Esch, Eur. J. Org. Chem. 2005, 3615-3631; c) R. G. Weiss, P. Terech, Molecular Gels: Materials with Self-Assembled Fibrillar Networks; Springer: New York, 2006; d) M. George, R. G. Weiss, Acc. Chem. Res. 2006, 39, 489497; e) G. C. Maity, J. Phys. Sci. 2007, 11, 156-171; f) E. Zaccarelli, J. Phys. Condens. Matter 2007, 19, 323101; g) S. Banerjee, R. K. Das, U. Maitra, J. Mater. Chem. 2009, 19, 6649-6687.

[3] a) D. Derossi, Y. Kajiwara, Y. Osada, Polymer Gels: Fundamentals and Biomedical Applications, Plenum Press, New York, 1991, and references therein; b) J. H. Jung, S. Shinkai, Top. Curr. Chem. 2004, 248, 223-260; c) N. M. Sangeetha, U. Maitra, Chem. Soc. Rev. 2005, 34, 821-836; d) R. V. Ulijn, A. M. Smith, Chem. Soc. Rev. 2008, 37, 664-675; e) M. O. M. Piepenbrock, G. O. Lloyd, N. Clarke, J. W. Steed, Chem. Rev. 2010, 110, 1960-2004.

[4] a) T. Tanaka, Sci. Am. 1981, 244, 110-116; b) Y. Osada, A. R. Khokhlov, Polymer Gels and Networks; Marcel Dekker: New York, 2002; c) S.-k. Ahn, R. M. Kasi, S.-C. Kim, N. Sharma, Y. Zhou, Soft Matter 2008, 4, 1151-1157.

[5] a) L. A. Estroff, A. D. Hamilton, Chem. Rev. 2004, 104, 1201-1218, and references therein; b) P. Xie, R. Zhang, J. Mater. Chem. 2005, 15, 2529-2550; c) M. Sangeetha, U. Maitra, Chem. Soc. Rev. 2005, 34, 821-836; d) M. George, R. Mathew, R. G. Weiss, Molecular Gels, 2006, 449-551; e) D. K. Smith, Chem. Commun. 2006, 34-44; f) D. J. Adams, Macromol. Biosci. 2011, 11, 160-173; g) A. Dawn, T. Shiraki, S. Haraguchi, S. Tamaru, S. Shinkai, Chem. Asian J. 2011, 6, 266-282; h) X. Yang, G. Zhang, D. Zhang, J. Mater. Chem. 2012, 22, 38-50.

[6] A. Ajayaghosh, V. K. Praveen, C. Vijayakumar, Chem. Soc. Rev. 2008, 37, 109122.

[7] a) S. K. Chandran, N. K. Nath, S. Cherukuvada, A. Nangia, J. Mol. Struct. 2010 968, 99-107; b) R. Custelcean, Chem. Commun. 2008, 295-307; c) J. Jr. Rebek, Chem. Commun. 2000, 637-643; d) Bogdan, A., Rudzevich, Y., Vysotsky, M.O., Böhmer, V. Chem. Commun. 2006, 2941-2952.

[8] M. Yamanaka, J. Incl. Phenom. Macrocycl. Chem. 2013, 77, 33-48, and references therein.

[9] a) R. P. Herrera, V. Sgarzani, L. Bernardi, A. Ricci, Angew. Chem. Int. Ed. 2005, 44, 6576-6579; b) G. Dessole, R. P. Herrera, A. Ricci, Synlett 2004, 2374-2378.

[10] See the Supporting Information for further details.

[11] X. Fan, R. Burton, Open Fuel Energy Sci. J. 2009, 2, 100-109.

[12] A. Takahashi, M. Sakai, T. Kato, Polym. J. 1980, 12, 335-341.

[13] I. Kapoor, E.-M. Schön, J. Bachl, D. Kühbeck, C. Cativiela, S. Saha, R. Banerjee, S. Roelens, J. J. Marrero-Tellado, D. D. Diaz, Soft Matter 2012, 8, 3446-3456.

[14] a) Z. Dzolic, K. Wolsperger, M. Žinić. New J. Chem. 2006, 30, 1411-1419; b) A. Brizard, R. Oda, I. Huc, Top. Curr. Chem. 2005, 256, 167-218.

[15] a) S. R. Jadhav, P. K. Vemula, R. Kumar, S. R. Raghavan, G. John, Angew. Chem. Int. Ed. 2010, 49, 7695-7698; b) S. Battacharya, Y. Krishnan-Ghosh, Chem. Commun. 2001, 185-186. 
[16] a) A. Prathap, K. M. Sureshan, Chem. Commun. 2012, 48, 5250-5252; b) S. Basak, J. Nanda, A. Banerjee, J. Mater. Chem. 2012, 22, 11658-11664; c) S Debnath, A. Shome, S. Dutta, P. K. Das, Chem. Eur. J. 2008, 14, 6870-6881.

[17] X. Yu, Y. Li, D. Wu, Z. Ma, S. Xing, New J. Chem. 2013, 37, 1201-1205.

[18] M. J. Kamlet, J. L. M. Abboud, M. H. Abraham, R. W. Taft, J. Org. Chem. 1983, 48, 2877-2887.

[19] M. Raynal, L. Bouteiller, Chem. Commun. 2011, 8271-8273.

[20] A. R. Hirst, D. K. Smith, Langmuir 2004, 20, 10851-10857.

[21] P. Fatás, J. Bachl, S. Oehm, A. I. Jiménez, C. Cativiela, D. D. Díaz, Chem. Eur. J. 2013, 19, 8861-8874.

[22] a) F. Allix, P. Curcio, Q. N. Pham, G. Pickaert, B. Jamart-Grégoire, Lanmui 2010, 26, 16818-16827; b) J. Makarevic, M. Jokic, Z. Raza, Z. Stefanic, B. Kojic-Prodic, M. Zinic, Chem. Eur. J. 2003, 9, 5567-5580.

[23] a) L. Frkanec, M. Zinic, Chem. Commun. 2010, 522-537; b) V. Caplar, M. Zinic J.-L. Pozzo, F. Fages, G. Mieden-Gundert, F. Vögtle, Eur. J. Org. Chem. 2004, 4048-4059.

[24] a) R. M. Osuna, V. Hernández, J. T. L. Navarrete, E. D’Oria, J. J. Novoa, Theor Chem. Acc. 2011, 128, 541-553; b) K. E. Riley, P. Hobza, J. Chem. Theor. Comput. 2008, 4, 232-242.

[25] R. E. Fischer, B. Tadic-Galeb, P. R. Yoder, Optical System Design, McGraw Hill, 2nd Ed., 2008.

[26] a) P. Kirilov, F. Gauffre, S. Franceschi-Messant, E. Perez, I. Rico-Lattes, $J$ Phys. Chem. B 2009, 113, 11101-11108; b) T. Peterfi, Arch. Entwicklungsmech. Organ. 1927, 112, 680-686.

[27] a) G. Mezger, The Rheology Handbook, Vincentz Network, Hannover, Germany, 2011; b) H. A. Barnes, J. Non-Newtonian Fluid Mech. 1997, 70, 1-33.

[28] a) M. Salman, Int. J. Life Sci. Pharm. Res. 2012, 2, 128-138; b) M.-O. M. Piepenbrock, N. Clarke, J. W. Steed, Soft Matter 2011, 7, 2412-2418.

[29] K. Patel, S. Kapoor, D. P. David, T. Mukherhjee, J. Chem. Sci. 2005, 117, 53-60.

[30] a) D. Boyer, P. Tamarat, A. Maali, B. Lounis, M. Orrit, Science 2002, 297, 1160-1163; b) Z. L. Wang, J. Phys. Chem. B 2000, 104, 1153-1175.

[31] A. Chakrabarty, U. Maitra, J. Phys. Chem. B. 2013, 117, 8039-8046.

[32] D. D. Diaz, D. Kuhbeck, R. J. Koopmans, Chem. Soc. Rev. 2011, 40, 427-448.
[33] J. Bachl, A. Hohenleutner, B. B. Dhar, C. Cativiela, U. Maitra, B. König, D. D. Díaz, J. Mater. Chem. A 2013, 1, 4577-4588.

[34] J. M. Roberts, B. M. Fini, A. A. Sarjeant, O. K. Farha, J. T. Hupp, K. A. Scheidt, J. Am. Chem. Soc. 2012, 134, 3334-3337.

[35] R. P. Herrera, D. Monge, E. Martín-Zamora, R. Fernández, J. M. Lassaletta, Org. Lett. 2007, 9, 3303-3306.

[36] M. P. Sibi, K. Itoh, J. Am. Chem. Soc. 2007, 129, 8064-8065.

[37] B. Procuranti, S. J. Connon, Chem. Commun. 2007, 1421-1423.

[38] S. W. Jeong, S. Shinkai, Nanotechnology 1997, 8, 179-183.

[39] a) K. E. Riley, M. Pitoňák, P. Jurečka, P. Hobza, M. J. Frisch, G. W. Trucks, H. B. Schlegel, G. E. Scuseria, M. A. Robb, J. R. Cheeseman, G. Scalmani, V. Barone, B. Mennucci, G. A. Petersson, H. Nakatsuji, M. Caricato, X. Li, H. P. Hratchian, A. F. Izmaylov, J. Bloino, G. Zheng, J. L. Sonnenberg, M. Hada, M. Ehara, K. Toyota, R. Fukuda, J. Hasegawa, M. Ishida, T. Nakajima, Y. Honda, O. Kitao, H. Nakai, T. Vreven, J. A. Montgomery, Jr., J. E. Peralta, F. Ogliaro, M. Bearpark, J. J. Heyd, E. Brothers, K. N. Kudin, V. N. Staroverov, T. Keith, R. Kobayashi, J. Normand, K. Raghavachari, A. Rendell, J. C. Burant, S. S. Iyengar, J. Tomasi, M. Cossi, N. Rega, J. M. Millam, M. Klene, J. E. Knox, J. B. Cross, V. Bakken, C. Adamo, J. Jaramillo, R. Gomperts, R. E. Stratmann, O. Yazyev, A. J. Austin, R. Cammi, C. Pomelli, J. W. Ochterski, R. L. Martin, K. Morokuma, V. G. Zakrzewski, G. A. Voth, P. Salvador, J. J. Dannenberg, S. Dapprich, A. D. Daniels, O. Farkas, J. B. Foresman, J. V. Ortiz, J. Cioslowski, and D. J. Fox, Gaussian 09, Revision C.01, Gaussian, Inc., Wallingford CT, 2010.

[40] Y. Zhao, D. G. Truhlar, J. Chem. Phys. 2006, 125, 194101.

[41] A. D. McLean, G. S. Chandler, J. Chem. Phys. 1980, 72, 5639-5648.

[42] K. Remya, C. H. Suresh, J. Comput. Chem. 2013, 34, 1341-1353.

[43] J. Tomasi, B. Mennucci, R. Cammi, Chem. Rev. 2005, 105, 2999-3094.

[44] S. F. Boys, F. Bernardi, Mol. Phys. 1970, 19, 553-566.

Received: ((will be filled in by the editorial staff))

Revised: ((will be filled in by the editorial staff)) Published online: ((will be filled in by the editorial staff)) 